Article

\title{
Estimation of the Electricity Storage Volume Density of Compact SMESs of a New Concept Based on Si Microfabrication Technologies
}

\author{
Tomoyoshi Motohiro ${ }^{1, *(D)}$, Minoru Sasaki ${ }^{2}$, Joo-hyong Noh $^{3}$ and Osamu Takai ${ }^{3}$ \\ 1 Institutes of Innovation for Future Society, Nagoya University, Furo-cho, Chikusa-ku, Nagoya 464-8603, Japan \\ 2 Toyota Technological Institute, Graduate School of Engineering, Hisakata 2-12-1, Tempaku-ku, \\ Nagoya 468-8511, Japan; mnr-sasaki@toyota-ti.ac.jp \\ 3 Materials \& Surface Engineering Research Institute, Kanto-Gakuin University, 1162-2, Ogikubo, \\ Odawara 250-0042, Japan; noh@kanto-gakuin.ac.jp (J.-h.N.); takai@kanto-gakuin.ac.jp (O.T.) \\ * Correspondence: tomoyoshi.motohiro@chem.material.nagoya-u.ac.jp or tomoyoshi.motohiro@gmail.com
}

Citation: Motohiro, T.; Sasaki, M.; Noh, J.-h.; Takai, O. Estimation of the Electricity Storage Volume Density of Compact SMESs of a New Concept Based on Si Microfabrication Technologies. Magnetochemistry 2021, 7, 44. https://doi.org/10.3390/ magnetochemistry7030044

Academic Editor: Cristina Favieres

Received: 22 February 2021

Accepted: 17 March 2021

Published: 23 March 2021

Publisher's Note: MDPI stays neutral with regard to jurisdictional claims in published maps and institutional affiliations.

Copyright: (c) 2021 by the authors. Licensee MDPI, Basel, Switzerland. This article is an open access article distributed under the terms and conditions of the Creative Commons Attribution (CC BY) license (https:/ / creativecommons.org/licenses/by/ $4.0 /)$.

\begin{abstract}
A compact superconducting magnetic energy storage system (SMES) produced by Si micro fabrication technologies has been proposed to improve electricity storage volume density, $w$, in the sub-Wh/L range of conventional SMESs and to produce them at a low cost by mass production. In parallel with the experimental development reported previously, a series of trials was performed to estimate a feasible value of $w$ based on the calculation of the magnetic field generated by the compact SMES by improving the calculation models step by step. In this work, the experimentally obtained magnetic flux density dependence of superconductive critical current density was taken into consideration for the first time in this series of trials, together with the additional improvement of the calculation models. The results of the estimation indicated that a compact SMES produced by the proposed concept can attain a $w$ in the Wh/L range or more, ranking with or surpassing that of presently used capacitors.
\end{abstract}

Keywords: SMES; capacitor; electricity storage volume density; critical current density; Si microfabrication technologies; $\mathrm{Si}$ wafer; spiral trench; electromagnetic stress; $\mathrm{NbN} ; \mathrm{YBa}_{2} \mathrm{Cu}_{3} \mathrm{O}_{7-\delta}$

\section{Introduction}

\subsection{Background and Motivation of the Research}

Capacitors are typical electronic passive components widely used in varieties of electronic circuits. Capacitors are fundamentally used for electricity storage as the energy of the electric field, which is expressed by Equation (1):

$$
u_{e}=\frac{1}{2} \varepsilon_{0} E^{2}
$$

where $u_{\mathrm{e}}, \varepsilon_{0}$, and $E$ stand for the energy density of the electric field, the permittivity of the vacuum, and the strength of the electric field. Electricity storage in capacitors is based on an electrostatic, a purely physical phenomenon. The benefit of this fact is the ability of rapid charge and discharge in comparison to rechargeable batteries based on electrochemical reactions. Many varieties of capacitors are commercially available, such as ceramic capacitors, polymer film capacitors, electrolytic capacitors, and electric doublelayer capacitors (EDLC). Among them, EDLCs are reported with a high electric energy storage density $(w)$. Judging from open websites, typical specifications for size, maximum stored electric energy, and $w$, are Specifications 1: $\varnothing 18 \mathrm{~mm} \times 70 \mathrm{~mm}, 0.1 \mathrm{Wh}, 5.7 \mathrm{Wh} / \mathrm{L}$ [1]; Specifications 2: $\varnothing 40 \mathrm{~mm} \times 150 \mathrm{~mm}, 1.2 \mathrm{Wh}, 6.5 \mathrm{Wh} / \mathrm{L}$ [2]; and Specifications 3: $\varnothing 51$ $\mathrm{mm} \times 142 \mathrm{~mm}, 2.2 \mathrm{Wh}, 7.5 \mathrm{Wh} / \mathrm{L}$ [3]. Here, it should be noted that what is called "hybrid energy storage capacitors" are reported to have a higher w, with Specifications 4 : 
$35 \mathrm{~mm} \times 25 \mathrm{~mm} \times 20 \mathrm{~mm}, 0.2-0.9 \mathrm{Wh}, 11-50 \mathrm{Wh} / \mathrm{L}$ [4]. However, they are excluded from the purely physical electric energy storage means based on Equation (1), because they are based not only on the electrostatic storage of energy but also on the electrochemical storage of energy.

As a counterpart of Equation (1), in electromagnetics, the energy of magnetic field $u_{\mathrm{m}}$ is formulated as Equation (2):

$$
u_{m}=\frac{1}{2} \mu_{0} H^{2}
$$

where $\mu_{0}$ and $H$ stand for the permeability of a vacuum and magnetic field strength, respectively. As for means of physical electric energy storage based on Equation (2), superconducting magnetic energy storage systems (SMES) were developed for power system control, such as load fluctuation compensation and power system stabilization, in which a superconducting coil generates a magnetic field [5]. A state-of-the-art practical example being in operation is of $5.6 \mathrm{kWh}[6,7]$, in which superconducting coils are formed by coiling composite wires composed of $\mathrm{NbTi}$ superconducting wires and $\mathrm{Cu}$ wires twisted together $(\mathrm{NbTi} / \mathrm{Cu}$ coils). $\mathrm{Cu}$ wires are employed to assume a role of stabilizer to homogenize the local temperature fluctuations of $\mathrm{NbTi}$ superconducting wires with the help of the high thermal conductivity of $\mathrm{Cu}$. The local temperature fluctuation can lead to quenching-that is, the accidental loss of superconductivity in $\mathrm{NbTi}$ wires. $\mathrm{Cu}$ wires also assume the role of the current bypass in the case of quenching. Because $\mathrm{NbTi} / \mathrm{Cu}$ composite wires are rigid and difficult to coil at a small curvature radius, the size of a container comprising superconducting $\mathrm{NbTi} / \mathrm{Cu}$ coils can be as large as $\varnothing 3.2 \mathrm{~m} \times 3.2 \mathrm{~m}[6,7]$. This gives a $w$ of about $0.18 \mathrm{Wh} / \mathrm{L}$ judging from the literature. However, ancillary facilities are also necessary in a SMES system including a cryogenic refrigeration apparatus and magnetic shields to prevent the intense magnetic field generated by the superconducting coils from causing false operation in peripheral electronic and mechanical apparatuses. Suppose the volume of these ancillary facilities is the same as that of the container of the superconducting magnet, the total volume is doubled, and the $w$ is estimated to be $0.09 \mathrm{Wh} / \mathrm{L}$. More recently, $\mathrm{YBa}_{2} \mathrm{Cu}_{3} \mathrm{O}_{7-\delta}(\mathrm{Y} 123)$ tape or $\mathrm{Bi}_{2} \mathrm{Sr}_{2} \mathrm{Ca}_{2} \mathrm{Cu}_{3} \mathrm{O}_{10+\delta}(\mathrm{Bi2223}) / \mathrm{Ag}$ tape has been used to fabricate a superconducting coil in place of the $\mathrm{NbTi} / \mathrm{Cu}$ composite wire, and the sizes of the superconducting magnet were much reduced [8,9]. As a typical example, the size of a coil with an electricity storage capacity of $278 \mathrm{Wh}$ was reportedly $\varnothing 0.78 \mathrm{~m} \times 0.69 \mathrm{~m}$, and the $w$ can be calculated to be $0.84 \mathrm{Wh} / \mathrm{L}$ judging from a recent, state-of-the-art report [10]. If ancillary facilities of the same volume are taken into consideration, the $w$ will be around $0.4 \mathrm{Wh} / \mathrm{L}$.

A superconducting current in a coil in the vicinity of the critical superconducting current density $\left(j_{c}\right)$ can induce an intense magnetic field, resulting in a huge electromagnetic force imposed on the superconducting current along the radial direction to enlarge the coil diameter ('hoop stress'). To prevent the coil from breaking under this force, the coil must be protected by specially designed reinforced structures [11-16]. The necessity of this reinforced structures is another factor that causes the large volumes of conventional SMES systems.

Therefore, despite using the same physical electric energy storage means based on similar expressions-Equations (1) and (2) - in electromagnetics, the sizes are much larger and the $w$ is much smaller in the present SMESs in operation, in comparison with commercially available and widely used capacitors. In addition to the lower $w$ in comparison with capacitors, SMESs are also inferior to capacitors in terms of their costs. This is partly caused by the fact that SMESs being in operation are made-to-order heavy electric machinery products, in contrast to mass-produced capacitors, which are commercially available with many choices. Therefore, the development of a mass-producible SMES will be an important way to expand use of electricity storage means based on Equation (2). Motivated by this, we proposed and pursued a novel approach to improve the $w$ and mass producibility of SMES. 


\subsection{A Novel Approach to Increase the $w$ and Mass Producibility of SMES}

To increase the $w$ of SMES from a sub-Wh/L level to a sub-10 Wh/L level ranking with the $w$ of capacitors or more, we proposed the concept of a compact SMES, as shown in Figure 1. Without winding the superconducting cables or tapes at a small curvature radius, a superconducting thin-film coil is deposited and embedded in a spiral trench to prevent it from coming off from the substrate by electromagnetic stress $S$, which is applied to the coil, as shown in Figure 1a. Because $S$ is a hoop stress put upon the outer wall of the trench, the superconducting current must be reduced to keep $S$ lower than $1 / 3$ of the mechanical strength limit of the Si wafer $\sigma_{\mathrm{Si}}: 4 \mathrm{GPa}$. Here, 1/3 is a safety factor. Multiple Si wafers, which are engraved with a spiral coil (hereafter, we call it a wafer coil for simplicity), are stacked and series-interconnected via through-holes based on 3D integration technology to form a cylindrical coil unit, as shown in Figure 1b [17]. In a typical possible concept design, the four units together with a compact cryogenic refrigerator are combined to form a typical possible minimum system, as shown in Figure 1c. Compact cryogenic refrigerators have been greatly improved recently and are on the market [18]. Here, the cryogenic refrigerator is drawn as a simplified form composed of a cuboid of $b \times b \times h$ and a cylindrical tower with a height of $h$.

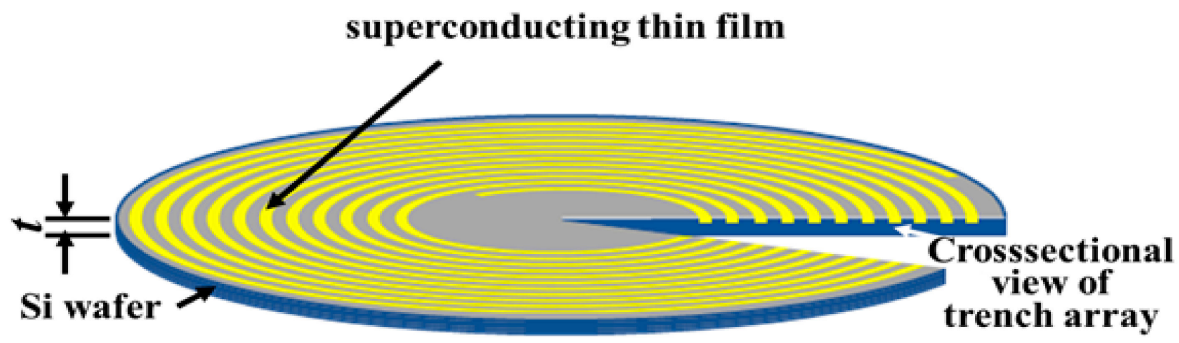

(a) Superconducting thin film coil embedded in a spiral trench on a Si wafer (wafer coil).

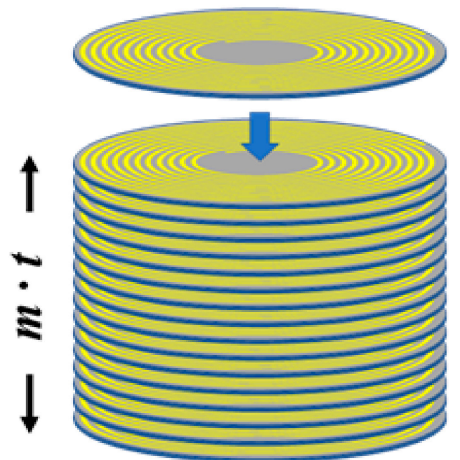

(b) A cylindrical coil unit made of a stack of seriesinterconnected wafer coils (wafer stack).

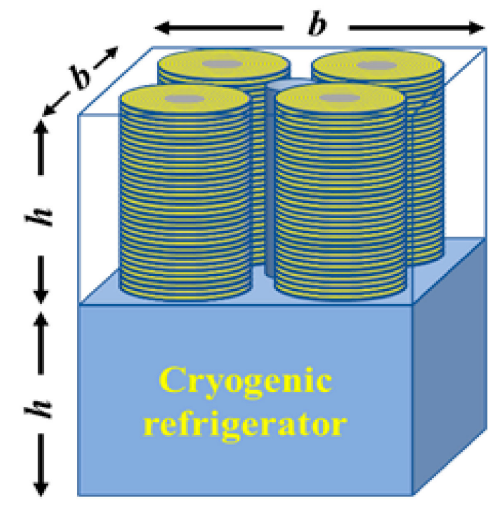

(c) A design of a compact SMES system composed of 4 cylindrical coil units with a cryogenic refrigerator.

Figure 1. Schematic representation of a typical possible minimum composition of the compact SMES based on the proposed concept. $t$ is the thickness of the Si wafer and $m$ is the number of stacked $\mathrm{Si}$ wafers. $b$ and $h$ represent the approximate external sizes of the composition. A special case of mboxemphh $\cong m \bullet t$ is drawn in (c).

\subsection{Experimental Proof of Concept Using Superconducting NbN Thin Films}

A proof-of-concept experiment was performed on a single Si wafer using reactively sputter-deposited $\mathrm{NbN}$ superconducting thin films [19] and an electrolytically plated $\mathrm{Cu}$ layer as a stabilizer. Figure 2a-e schematically displays the process of trench formation; deposition of $\mathrm{NbN}$ and $\mathrm{Cu}$; and the removal of unnecessary deposits of $\mathrm{NbN}$ and $\mathrm{Cu}$, except for those in the trench, by chemical-mechanical polishing (CMP) step by step. Figure $2 \mathrm{f}-\mathrm{h}$ 
schematically displays an example of the wafer bonding and series interconnection processes. Details of these processes were described in our previous report [20,21]. Figure 3a,b shows two photos of the Si wafer coils in a preliminary fabrication, corresponding to the process steps shown in Figure $2 \mathrm{~d}$,e, respectively. These process technologies were used to fabricate normal conductive MEMS (microelectromechanical system) inductors on Si wafers [22]. The measurement of the current-voltage characteristics in cryogenic temperatures together with the measurement of magnetic field by a gauss meter showed an energy storage of $0.01 \mathrm{~mJ}$ [20]. Increasing the $\mathrm{NbN}$ thickness by mitigating the film stress [23], the stored energy increased up to $0.1 \mathrm{~mJ}$ [24]. Here, the experimentally obtained $j_{\mathrm{c}}$ of $\mathrm{NbN}-1100 \mathrm{~A} / \mathrm{mm}^{2}$ - was used [19]. The largest $S$ near the central axis of the coil unit, $S_{\text {max }}$, estimated from the magnetic flux density $B$ along the central axis of a single-layer solenoid was $0.11 \mathrm{GPa}$, which was well below $\sigma_{\mathrm{Si}} / 3[20,24]$.

(a) Schematical cross-sectional view of unprocessed Si wafer

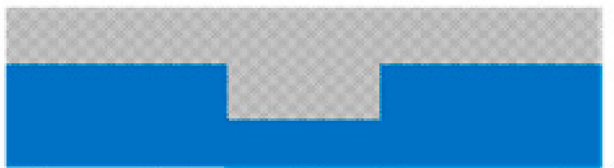

(b) Trench formation by repeated reactive ion etching process

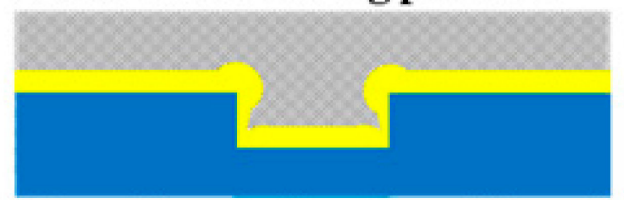

(c) Reactive sputter-deposition of superconducting $\mathrm{NbN}$ films

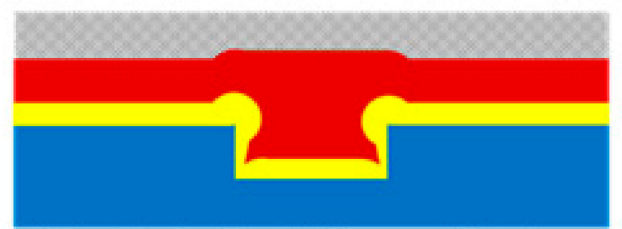

(d) Electrolytic plating of Cu layer as a stabilizer

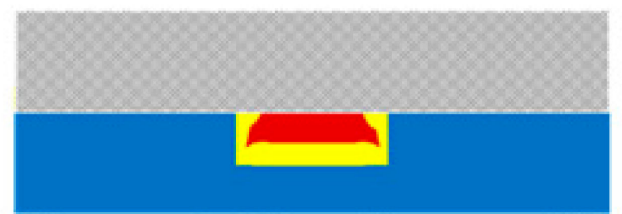

(e) Removal of deposits except those in the trench by Chemical Mechanical Polishing

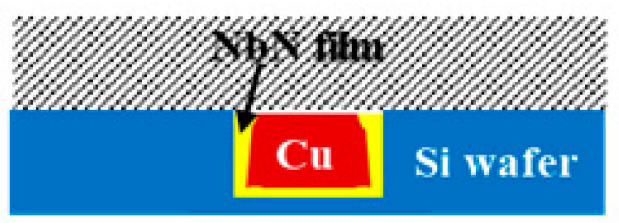

(f) Schematical cross-sectional view of superconducting $\mathrm{NbN}$ film and Cu stabilizer in the trench

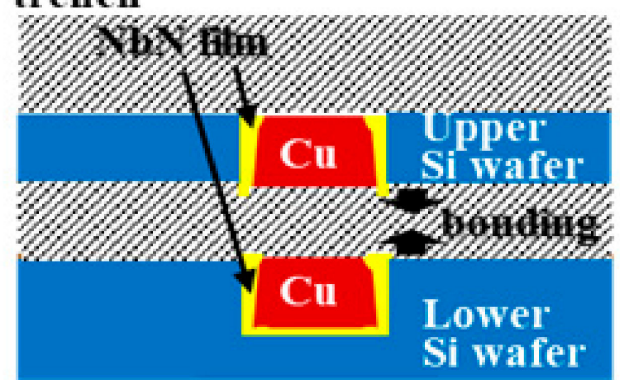

(g) Exposure of the NbN film and plated $\mathrm{Cu}$ by etching the back side of the upper Si wafer

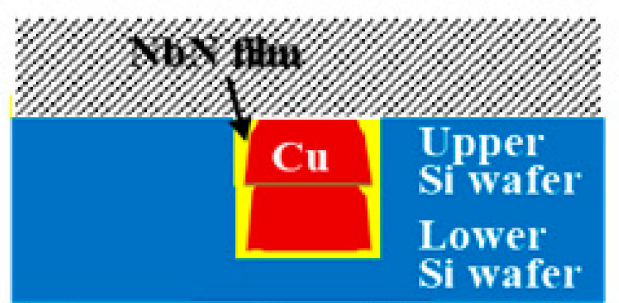

(h) Interconnection of the NbN films and plated $\mathrm{Cu}$ of the upper and the lower $\mathrm{Si}$ wafers by $\mathrm{Si}$ wafer bonding process

Figure 2. (a-e) The processes of trench formation; the deposition of $\mathrm{NbN}$ and $\mathrm{Cu}$; and the removal of unnecessary deposits of $\mathrm{NbN}$ and $\mathrm{Cu}$, except for those in the trench, by chemical-mechanical polishing (CMP). (f-h) Examples of the processes of the wafer bonding and series interconnection of the wafer coils. 


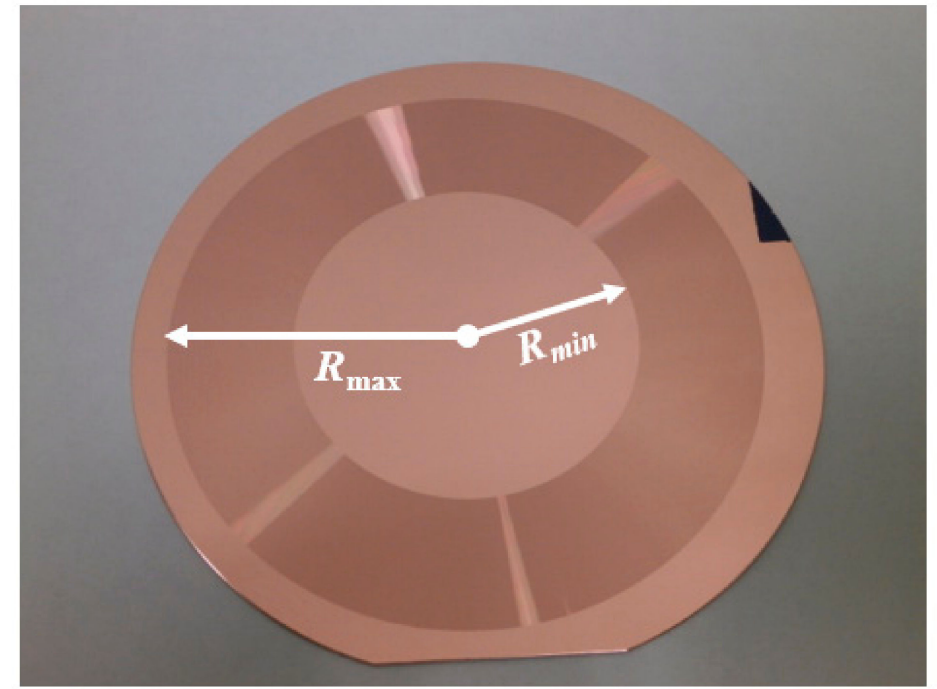

(a)

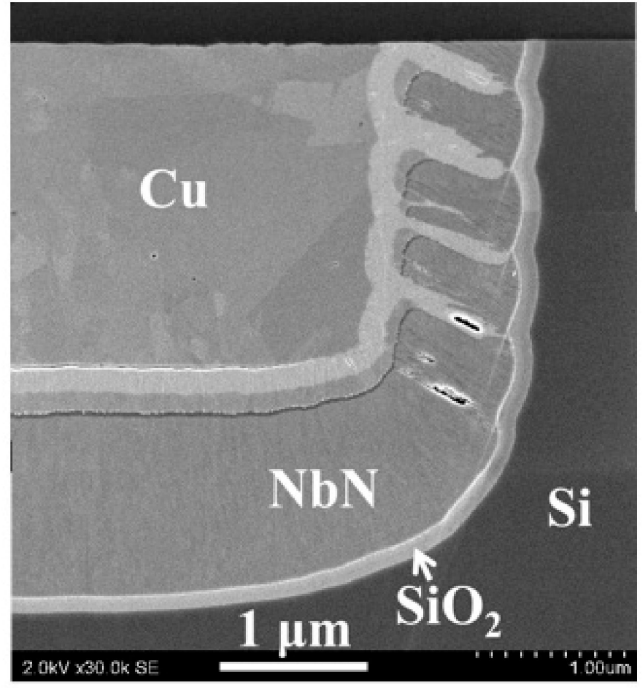

(b)

Figure 3. (a) A $101.6 \mathrm{~mm} \mathrm{Si} \mathrm{wafer} \mathrm{coil} \mathrm{after} \mathrm{the} \mathrm{electrolytic} \mathrm{plating} \mathrm{of} \mathrm{Cu}$ corresponding to Figure $2 \mathrm{~d}$. $R_{\max }$ is the radius of the outer trench wall of the outermost trench and $R_{\min }$ is the radius of the inner trench wall of the innermost trench. (b) Cross-sectional SEM photograph of a half of the trench with an $\mathrm{NbN}$ sputter-deposited layer and electrolytically plated $\mathrm{Cu}$ after $\mathrm{CMP}$, corresponding to Figure $2 \mathrm{e}$.

\subsection{Replacement of $\mathrm{NbN}$ by $\mathrm{YBa}_{2} \mathrm{Cu}_{3} \mathrm{O}_{7-\delta}$ to Increase Electricity Storage Volume Density}

For further improvement, we moved on to the replacement of the reactively sputterdeposited $\mathrm{NbN}$ thin film by Y123 deposited by the metal-organic deposition (MOD) method [25-27]. Because metal-organic precursors dissolved in a carrier fluid such as propionic acid can easily go into the trench, this is advantageous in comparison with sputter deposition with a limited throwing power. Because the superconducting transition temperature $\left(T_{c}\right)$ of $Y 123$ is $90 \mathrm{~K}$, this replacement enables the use of liquid hydrogen at $20.3 \mathrm{~K}$ or even of liquid nitrogen at $77 \mathrm{~K}$ as a refrigerant instead of a cryogenic refrigerator to cool below $13 \mathrm{~K}$ in the case of $\mathrm{NbN}$. However, the real benefit of this replacement for SMES is that the $j_{\mathrm{c}}$ of $\mathrm{Y} 123$ can be much higher than that of $\mathrm{NbN}$ if we keep using a $4 \mathrm{~K}$ cryogenic refrigerator.Figure 4 displays two curves, (a) and (b), of the measured $j_{\mathrm{c}}$ of the c-axis-oriented $\mathrm{Y} 123$ thin-film tape at $4 \mathrm{~K}$ as a function of the magnetic flux density applied in parallel with the c-axis (redrawn from the literature [28-30]). According to curve (a), $j_{c}$ was measured to be up to $31 \mathrm{~T}$ in the applied magnetic flux density. There is no indication of a rapid decrease in $j_{\mathrm{c}}$ due to the accession of the critical magnetic flux density $B_{\mathrm{c}}$. It was also reported that $B_{\mathrm{c}}$ was also measured to be as high as $120 \mathrm{~T}$ at $4 \mathrm{~K}$ and continuously decreased with the temperature to $0 \mathrm{~T}$ at the critical temperature: $T_{\mathrm{c}}=90 \mathrm{~K}$ [31]. Curve (b) shows more modest values of $j_{\mathrm{c}}$ than the curve (a). Although the reported measured maximum applied magnetic field density was around $18 \mathrm{~T}$ in curve (b), it will be reasonable to conjecture that curve (b) in Figure 4 can be smoothly extrapolated beyond $18 \mathrm{~T}$ to at least $50 \mathrm{~T}$, as shown in curve (c). Typically, the values of $j_{\mathrm{c}}$ for the applied magnetic field of $15 \mathrm{~T}$ on curves (a) and (b) are $5 \times 10^{4} \mathrm{~A} / \mathrm{mm}^{2}$ and $2 \times 10^{4} \mathrm{~A} / \mathrm{mm}^{2}$, which are about 45.5 times and 10 times of $1100 \mathrm{~A} / \mathrm{mm}^{2}$ in the case of $\mathrm{NbN}$, respectively. Because $w$ is proportional to the square of the current, we may expect, in comparison to the case of $\mathrm{NbN}$, a $45.5 \times 45.5=2070$, and $18 \times 18=324$ times larger $w$ in the case of Y123 for curves (a) and (b), respectively. Here, it should be noted that the maximum magnetic flux density at the innermost trench, $B_{\max }$ is proportional to the superconducting current $I$, and $S$ is proportional to $I^{2}$. This means that the maximum superconducting current $I_{\max }$ in the coil must be much reduced from the value derived from $j_{\mathrm{c}}$ because of the limitation of $B_{\mathrm{c}}$ and $S=\sigma_{\mathrm{Si}} / 3$. We intuitively know that we can mitigate this large $B$ and $S$ applied to the innermost portion of the coil by making the radius of the inner wall of the innermost trench, 
$R_{\text {min }}$, larger, leaving the central part of the wafer uncovered with trenches, as shown in Figure 3a. However, we lose $w$ simultaneously. Therefore, we must estimate how much we could mitigate $B$ and $S$ at the innermost portion of the coil and how much we lose $w$ quantitatively in concrete designs of the wafer coils.

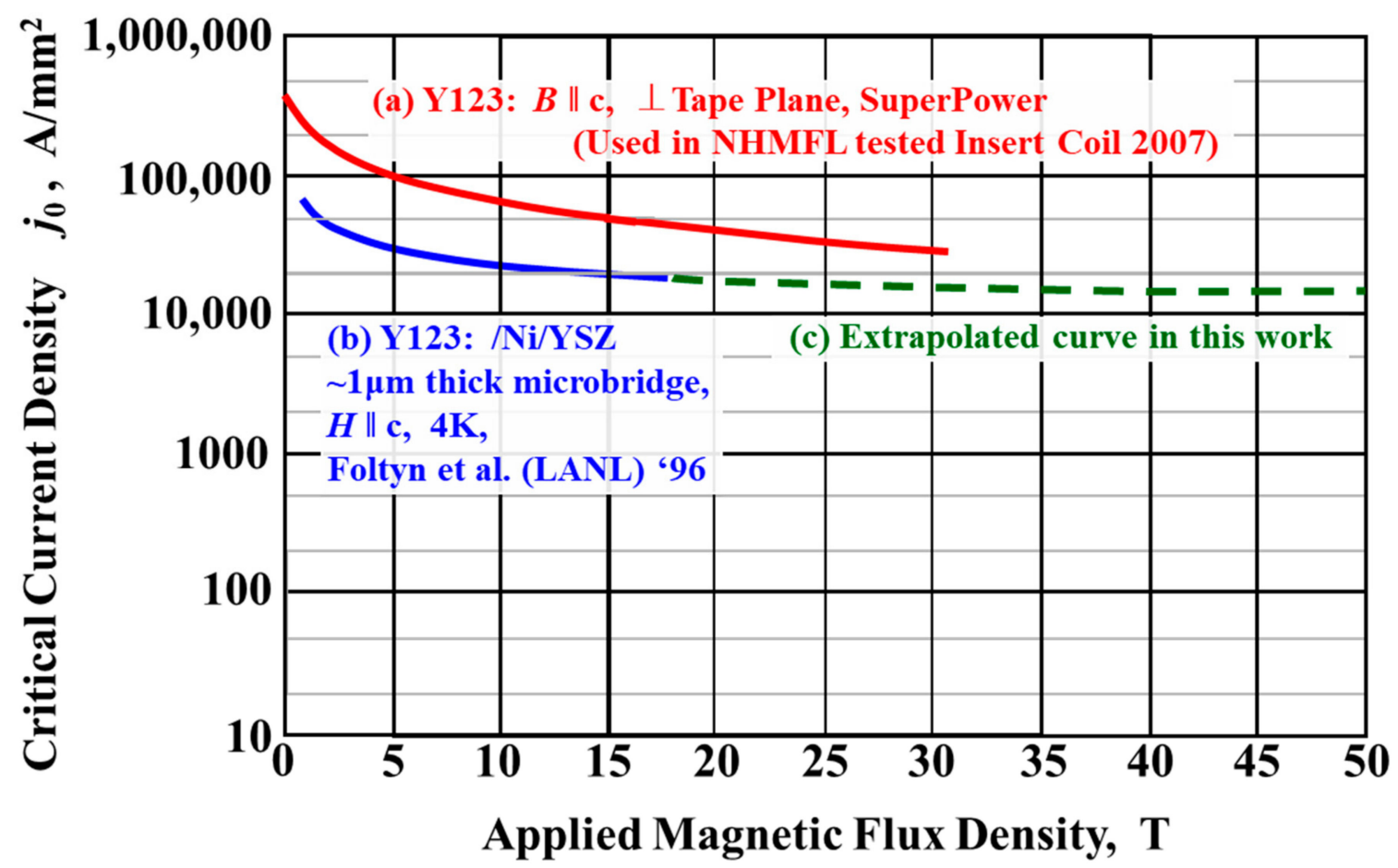

Figure 4. Experimentally obtained dependences of $j_{c}$ on the magnetic flux density in $Y 123$ thin films reported in $[28,29](\mathbf{a})$ and in [30] (b). (c) A supposed extended curve of (b) beyond $18 \mathrm{~T}$.

\subsection{Aim of This Work}

In our previous papers $[32,33]$, we reported our series of trial estimations on this subject under the fixed $j_{\mathrm{c}}$ of $2 \times 10^{4} \mathrm{~A} / \mathrm{mm}^{2}$. This value corresponds to a $j_{\mathrm{c}}$ at $15 \mathrm{~T}$ on line (b) in Figure 4. However, $j_{c}$ is dependent on $B_{\max }$, as shown in curves (b) and (c) in Figure 4. There is also experimental evidence of higher $j_{\mathrm{c}}$, as shown in curve (a) in Figure 4. To make a more realistic estimation, the $B_{\max }$ dependences of $j_{\mathrm{c}}$ based on the two different $j_{\mathrm{c}}-B_{\max }$ curves shown in Figure 4 were taken into consideration for the first time in our series of trial estimations. There was another item to refine the estimation. In the previous papers, the inductance $L$ of the cylindrical coil unit made of the stack of seriesinterconnected wafer coils was calculated as an equivalent single-solenoid coil, which generated the same magnetic field intensity at the center of the coil. As for the radius of the equivalent single-solenoid coil, we simply used a mean radius $a^{\prime}$ of $R_{\min }$ and the radius of the outer wall of the outermost trench of the wafer coil, $R_{\max }$-that is, $a^{\prime}=\left(R_{\max }+R_{\min }\right) / 2$. More precisely, the effective radius of the equivalent single-solenoid coil, $a$, should be smaller than $a^{\prime}$ because the contribution of an inner coil to the magnetic field at the center of the wafer coil is larger than that of an outer coil. In this work, the effective radius $a$ was also taken into consideration for the first time in our series of trial estimations. The aim of this work is to elucidate whether or not it is possible for SMES to increase $w$ to rank with and to surpass the $w$ of capacitors by the improved estimations. Tentatively, we started with the more modest curve (b) for the magnetic flux density dependence of $j_{\mathrm{c}}$. 


\section{Method of Estimation}

\subsection{Calculation of Magnetic Flux Density in a Wafer Coil}

Figure 5 schematically illustrates approximately one-half of the cross-section of a wafer coil, showing the principal design parameters of the spiral trench. Here, $R_{\text {wafer }}$ and $t$ stand for the radius and thickness of the Si wafer, respectively. Parameters $d, z$, and s stand for the trench width, trench depth, and trench wall thickness, respectively. The yellow rectangles indicate cross-sections of the trenches filled with Y123. Here, the trenches are supposed to be filled with Y123 and the presence of stabilizer layers as shown in Figures 2 and 3b is neglected for simplicity. The red circle with an arrow around a trench schematically indicates a typical magnetic field generated by the currents flowing Y123 in the trench from the back to the surface of the plane of paper.

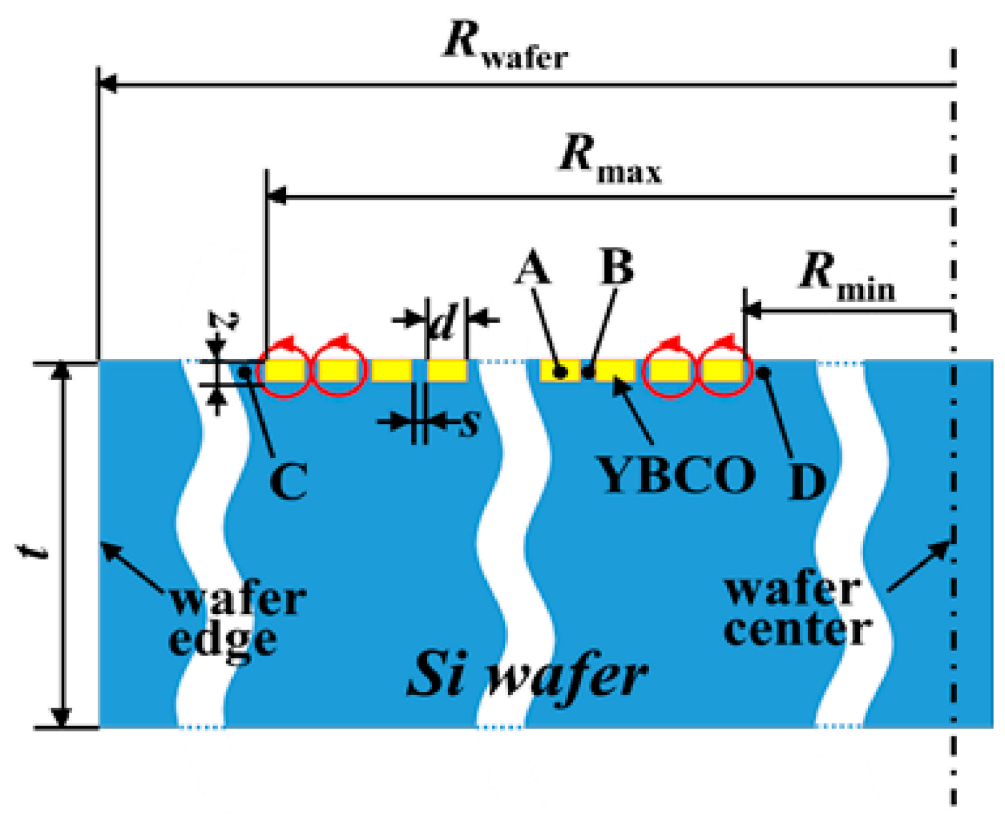

Figure 5. Schematic illustration of approximately one half of the cross-section of a wafer coil design in a Si wafer of $2 R_{\text {wafer }}$ diameter and $t$ thickness. Notations: $d, s, z, R_{\min }$, and $R_{\max }$ stand for the trench width, the trench wall thickness, the trench depth, the radius of the inner wall of the innermost coil, and the radius of the outer wall of the outermost coil, respectively. The yellow rectangles indicate cross-sections of the trenches filled with Y123.

As for the calculation of the distribution of magnetic flux density in the wafer coil, we replaced a spiral coil with the number of turns $n$ by $n$ conductive concentric circular current paths with the same $R_{\max }, R_{\min }, d, s$, and $z$ for a calculation model. In all the $n$ concentric current paths, the currents were supposed to be the same. The calculation was based on the Biot-Savart law, as shown in the following equation in SI units:

$$
B(r)=\frac{\mu_{0}}{4 \pi} \oint \frac{I(d q \otimes r \prime)}{|r \prime|^{3}}
$$

Here, $\mu_{0}$ is the magnetic permeability of free space: $4 \pi \times 10^{-7} \mathrm{H} / \mathrm{m} . I$ is the absolute value of the current in the circular current path. $B(r)$ is the vector for magnetic flux density at the position defined by vector $r$. The wire element $\mathrm{d} q$ is the vector of the circular current path element whose magnitude is the length of the differential element of the circular current path in the direction of the current at the position defined by vector $q$. The vector $r^{\prime}=r-q$ is the full displacement vector from $\mathrm{d} \boldsymbol{q} \mathrm{d} \ell\{\backslash$ displaystyle $\mathrm{d}\{\backslash$ boldsymbol $\{\backslash$ ell $\}\}$ at point $\boldsymbol{q} \ell\{\backslash$ displaystyle $\{\backslash$ boldsymbol $\{\backslash$ ell $\}\}\}$ to point $r$. The expression $\mathrm{d} \boldsymbol{q} \otimes r^{\prime}$ represents the cross product of the vectors $\mathrm{d} \boldsymbol{q}$ and $\boldsymbol{r}^{\prime}$. Numerical calculation was performed by integrating current density $j_{\mathrm{c}}$ in the $d$ by $z$ rectangular cross-section of the trench using 
the "integ.tplquad" function in a Python 3.6 calculation code.r $\{\backslash$ displaystyle $\backslash$ mathbf $\{\mathrm{r}\}\}$ The calculated magnetic flux density using this calculation code at the center of a single circular current normal to the plane of the circle was confirmed to agree with the value obtained from the analytical expression (4) deduced from Equation (3):

$$
B(z)=\frac{\mu_{0} \cdot I}{2 a_{1}}
$$

where $a_{1}$ is the radius of the circular current $I . B(r)$ in the trench in which $r$ can be equal to $q$ cannot be calculated by Equation (3) because the integrand containing $\boldsymbol{r}^{\prime}=\boldsymbol{r}-\boldsymbol{q}$ in its denominator reaches an infinite value. Typical calculations of $\boldsymbol{B}(\boldsymbol{r})$ for a single planar spiral coil formed in a spiral trench of width: $d=50 \mu \mathrm{m}$; depth: $z=30 \mu \mathrm{m}$; the trench wall thickness: $s=22 \mu \mathrm{m} ; R_{\min }=19 \mathrm{~mm}$; and turn number $n$ : 400 (resulting in $R_{\max }=47.8 \mathrm{~mm}$ on a $\mathrm{Si}$ wafer of the diameter $2 R_{\text {wafer }}=101.6 \mathrm{~mm}$ ) were performed assuming a fixed $j_{\mathrm{c}}$ of $2 \times 10^{4} \mathrm{~A} / \mathrm{mm}^{2}$. In the actual calculation by this calculation code, the $B(r)$ s caused by concentric 400 circular currents were summed up as a model of the single planer spiral coil. Figure 6a shows the results of the calculations of the radial distribution of the magnetic flux density normal to the wafer coil, $B_{z}(r)$, at the half depth of the trench. Here, the scalar: $r$ is the absolute value of the vector $r$ when the origin of $r$ and $\boldsymbol{q}$ is located at the center of the wafer plane. The calculation of $B_{\mathrm{Z}}(r)$ when $r$ is located out of the trench can be performed because $r^{\prime}$ in the denominator of the integrand in Equation (3) cannot be zero. Here, let us call this type of calculation Type I. The solid black circles stand for the calculated $B_{Z}(r)$ of Type I. We need to know $B_{Z}(r)$ penetrating the Y123 in the trench to estimate the electromagnetic hoop stress imposed on the Y123 as well as the $j_{\mathrm{c}}$ under the magnetic field generated by the other parts of the concentric circular currents. Because the magnetic flux generated by the current in a portion of a trench does not penetrate the same portion of the trench, the current in the corresponding portion can be eliminated from the estimation of $B_{z}(r)$. Accordingly, the calculation of $B_{z}(r)$ in the trench was also performed by eliminating the current element in a small volume portion of the trench surrounding the calculating position $r$ from the integral shown in Equation (3) so that $r$ does not become equal to $q$. Let us call this type of calculation Type II. The open black circles in Figure 6a indicate the $B_{\mathrm{Z}}(r)$ calculated in the trench by Type II. The small volume portion was defined as the volume portion in the circular trench within the plane angle area $\pm \delta$ in the vicinity of the point $r$ subtended at the center of the $\mathrm{Si}$ wafer. Figure $6 \mathrm{~b}$ schematically display the small volume portion of a trench surrounding a calculated position indicated by $\mathbf{A}$ employed in the calculation of Type II in a special case where the trench is the inner-most circular trench. As for the concrete value of $\delta$ in Type II calculations, 0.001 radian was employed. In this sense, the calculation of Type I can be regarded as the calculation of Type II at $\delta=0$, as shown in Figure 6a. In this special case of the Type II calculation, several values of $B_{\mathrm{Z}}(r)$ for $\mathrm{r}<R_{\min }$, out of the trench, were also calculated at the several points including $r=0 \mathrm{~mm}$ and plotted with open black circles in Figure 6a. In the region $r<R_{\min }$, the points calculated by Type I and Type II are on the same line. Thus, it is duly elucidated that the effect of the elimination of the current in the volume portion of the innermost circular trench on $B_{\mathrm{z}}(r)$ in this area, $r<R_{\min }$, was negligible. When $r$ approached $R_{\min }$ in the area $r<R_{\min }$ at the position $\mathbf{D}$ shown in Figure $5, B_{\mathrm{Z}}(r)$ steeply increased in the Type I calculation. Figure 7 displays the calculated values of $B_{\mathrm{Z}}(r)$ more in detail than Figure 6a in the vicinity of the innermost trench. Type I calculations were only performed for the positions out of the trench, as plotted with the solid black circles (a), while Type II calculations were performed also in the trench, as plotted with the open black circles (c). Type I calculations for a single innermost circular trench without the other concentric trenches were also conducted and plotted with solid black triangles (b). It is obviously known that the increase in (a) when $r$ decreased and approached $R_{\min }=-19.00 \mathrm{~mm}$ was caused by the contribution of (b). In the Type II calculation, $B_{Z}(r)$ takes a smaller value of $0.71 \mathrm{~T}$ at position $\mathbf{A}(r=19.025 \mathrm{~mm})$ in the center of the trench in Figure $6 \mathrm{~b}$ in comparison with $0.84 \mathrm{~T}$ by the Type I calculation at $r=18.99 \mathrm{~mm}$. In the peripheral area near the edge of the Si wafer, as indicated with C 
in Figure 5, the values indicated with the solid black circles in Figure 6a steeply decrease with the distance from the wafer center, in contrast to the open black circles staying near the zero magnetic field line in the same figure. This is because the magnetic flux density produced by the outermost ring current gives negative $B_{z}(r)$ at the position $C$, while this does not take place in Type II calculation. Except for this difference, the calculated values for $B_{\mathrm{z}}(\mathrm{r})$ generally showed a decrease with the increase in $r$ for both the solid black circles and the open black circles in Figure 6a. As shown in Figure $6 a, B_{z}(r)$ takes the highest value in the innermost trench in a wafer coil.



Distance from the center of Si wafer: $r(\mathrm{~mm})$

(a)

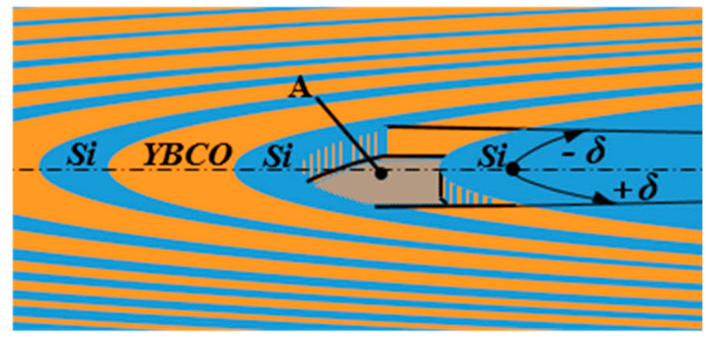

(b)

Figure 6. (a) Radial distribution of the calculated magnetic flux density $B_{Z}(r)$ normal to the wafer coil on the surface of the Si wafer of $2 R_{\text {wafer }}=101.6 \mathrm{~mm}$ and $t=0.5 \mathrm{~mm}$. The principal design parameters of the spiral coil, $d, s, z, R_{\max }, n$, and $R_{\min }$ are indicated in the inserts of the figure. (b) The volume portion within $\pm \delta$ radian removed in the circular trench from the integration in Equation (3) to calculate $B_{\mathrm{Z}}(r)$ at position A at the half width and at the half depth in the trench so that $r$ does not become equal to $q$.

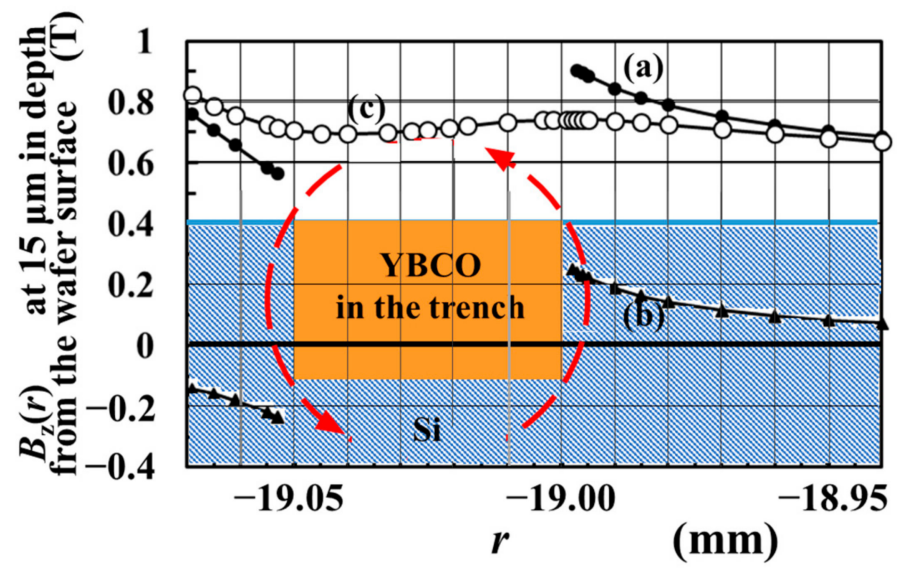

Figure 7. Calculated values of $B_{z}(r)$ in the vicinity of the innermost trench. (a) By Type I, (b) by Type I for a single circular trench, and (c) by Type II.

\subsection{Calculation of Magnetic Flux Density in a Stack of Wafer Coils}

In the case of the cylindrical unit of the stacked wafer coils, the magnetic flux density caused by a different wafer coil in the stack is also imposed on the innermost trench in question. Figure 8a schematically illustrates the component of the magnetic flux density $B_{\mathrm{Z}}(r)$ normal to the wafer at the innermost portion of the spiral coil at the radius $R_{\min }+d / 2$ and at depth $z / 2$ in a wafer caused by another wafer coil at a distance $x$ in the same stack 
with a red arrow. If the sum of contributions of magnetic flux densities caused by all the stacked wafer coils at a position in the innermost trench of a wafer coil exceeds $j_{c}$, the current density must be reduced to maintain superconductivity. The radial outwarddirected black arrow indicates the electromagnetic hoop stress imposed on the inner-most trench by the accumulated magnetic flux density of the all the contributions from the stacked wafer coils shown in the red arrows. Figure $8 \mathrm{~b}$ shows the dependence of $B_{\mathrm{z}}(r)$ on $x$ when $R_{\max }=47.8 \mathrm{~mm}, R_{\min }=19.0 \mathrm{~mm}, d=50 \mu \mathrm{m}, s=22 \mu \mathrm{m}, z=30 \mu \mathrm{m}$, and $t=0.5 \mathrm{~mm}$. Because $B_{\mathrm{z}}(r)$ is plotted in logarithmic scale, it is clearly shown that $B_{\mathrm{z}}(r)$ decreases rapidly with increasing $x$, and it is lower than $10 \%$ of the peak value when $x>2.5 \mathrm{~cm}$. The sum of the contributions $B_{\mathrm{Z}}(r)$ of all the wafers in a stack is denoted by $\Sigma B_{\mathrm{Z}}(r)$. Figure 9 displays a variation in $\Sigma B_{Z}(r)$ as a function of the position of the wafer $x$ in the stack in a typical case of the height of the cylindrical coil unit: $m=600$. Although $\Sigma B_{\mathrm{Z}}(r)$ has a maximum at $x=15 \mathrm{~cm}$, it is almost constant, except for the regions near $x=0 \mathrm{~cm}$ and $x=30 \mathrm{~cm}$. This is because the major contributions of $B_{\mathrm{Z}}(r)$ are of wafer coils at $x<2.5 \mathrm{~cm}$, as shown in Figure $8 \mathrm{~b}$. Figure 10a displays the dependence of the $\Sigma B_{z}(r)-x$ curve on $R_{\min }$. The maximum value of $\Sigma B_{z}(r)$ at $x=15 \mathrm{~cm}, B_{\text {max }}$,almost linearly decreased with $R_{\min }$, as shown in Figure 10b.

(a)

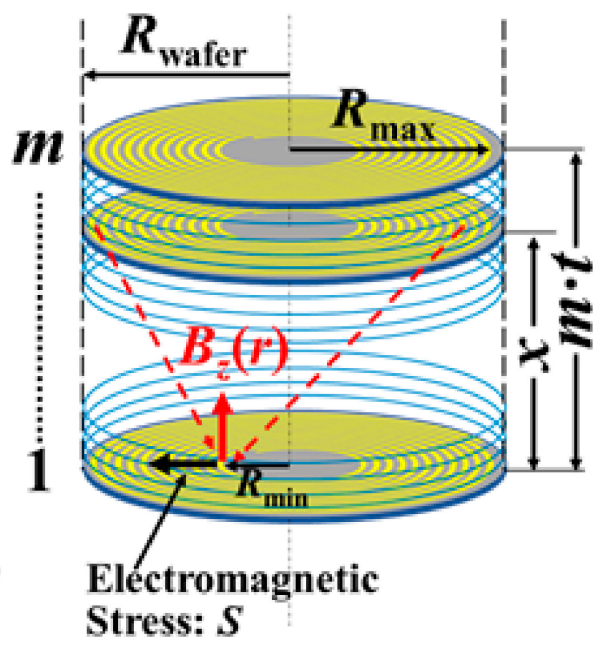

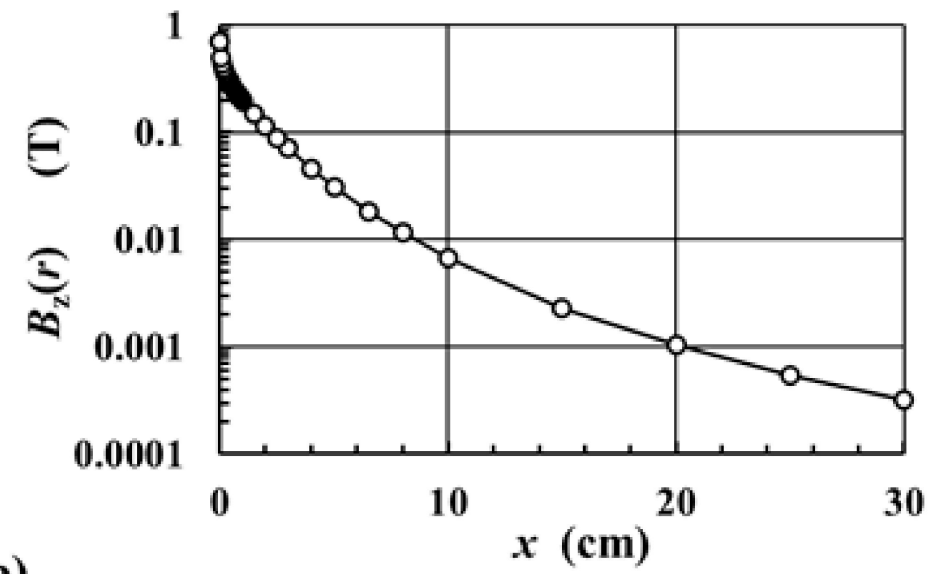

(b)
30

Figure 8. (a) Schematic illustration of the magnetic flux density in the innermost trench of a wafer coil indicated with a red arrow caused by a different wafer coil stacked above at $x$. Alongside, the generated electromagnetic hoop stress is also indicated by a radial outward-directed black arrow. (b) Dependence of the magnetic flux density $B_{\mathrm{Z}}(\mathrm{r})$ on $x$.

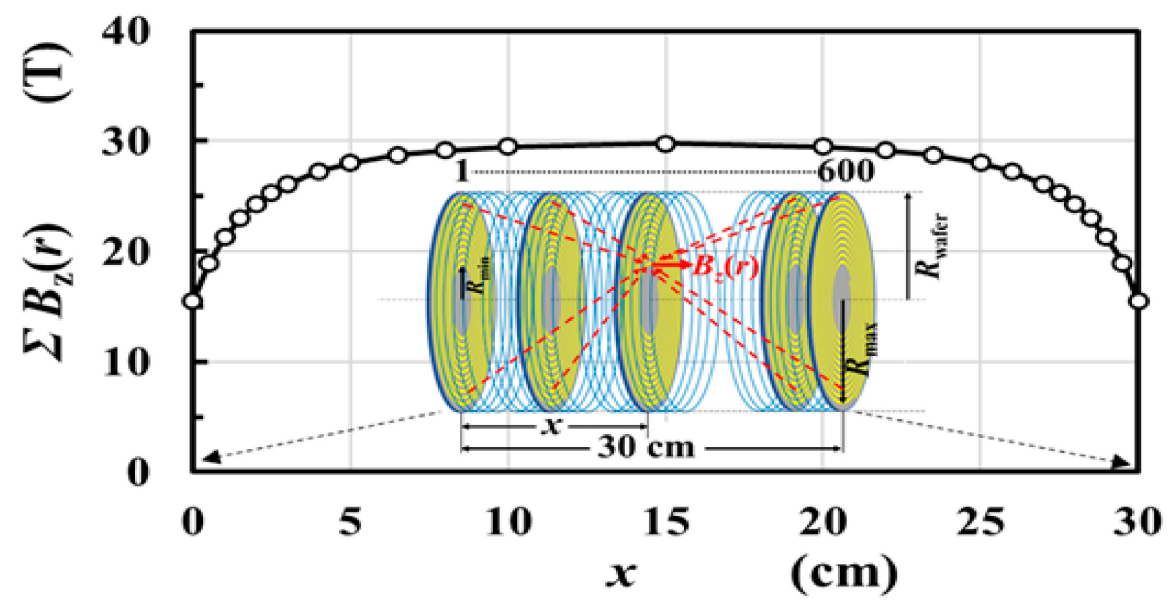

Figure 9. The sum of the contributions, $B_{\mathrm{z}}(r)$, of all the wafers in a stack, $\Sigma B_{\mathrm{z}}(r)$, as a function of the position of the wafer $x$ in the stack in a typical case of $m=600 . R_{\min }=19.0 \mathrm{~mm}$. 

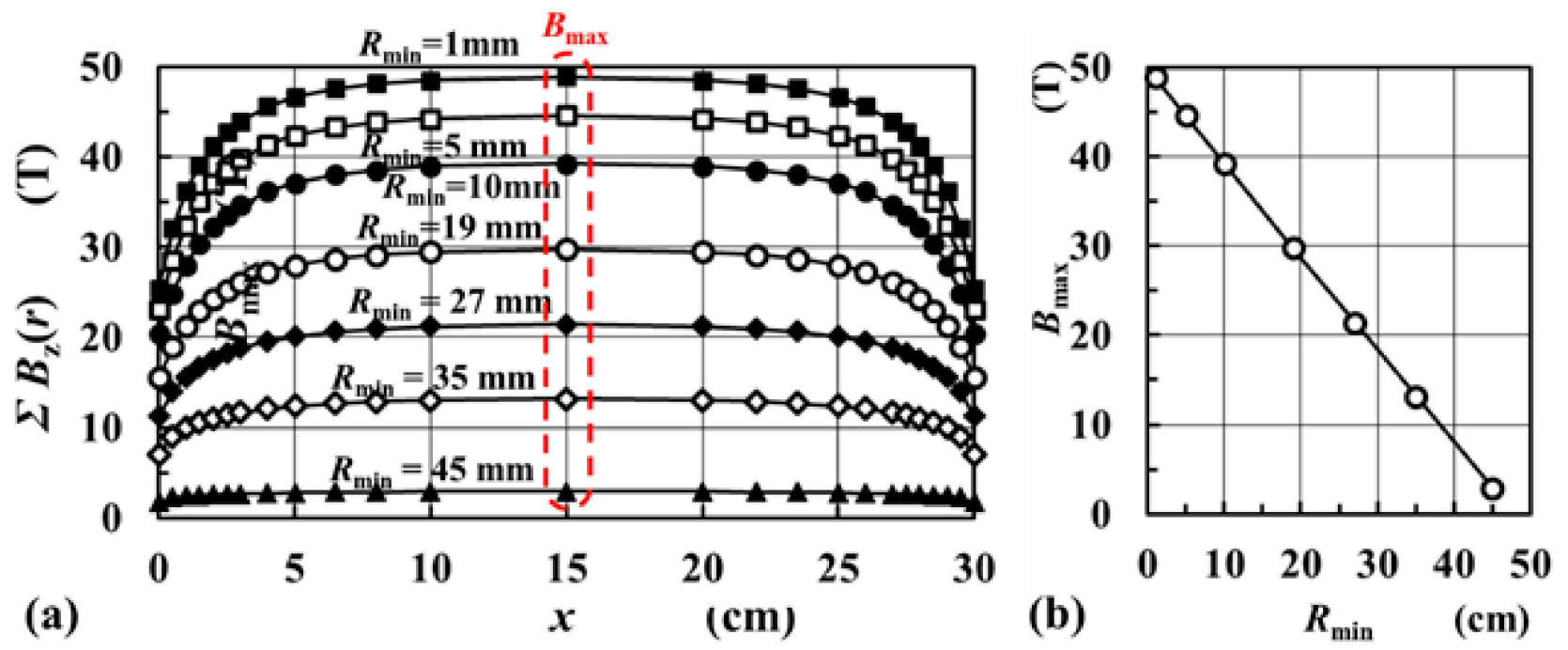

Figure 10. (a) Variation in $\Sigma B_{\mathrm{Z}}(r)-x$ profiles with $R_{\min }$ (b) Variation in $B_{\max }$ : the maximum value $\Sigma B_{\mathrm{Z}}(r)$ at $x=15 \mathrm{~mm}$ with $R_{\min }$.

\subsection{Calculation of Inductance of the Wafer Coil Stack}

$B_{\mathrm{Z}}(0)$-i.e., $B_{\mathrm{Z}}(r)$ at $r=0.0 \mathrm{~mm}$-was calculated as a function of $R_{\min }$, as shown in Figure 11. Here, the Type I calculations were employed because $r=0.0 \mathrm{~mm}$ represents the center of the wafer, which is located out of the trenches. The radius $a$ of a single circular current: $n \cdot d \cdot z \cdot j_{c}$, which yields the same magnetic flux density as $B_{Z}(0)$ at its center is calculated by Equation (4) and also plotted as a function of $R_{\min }$ in Figure 11. It is shown that $a$ is generally smaller than the mean radius of the spiral coil $a^{\prime}=\left(R_{\max }+R_{\min }\right) / 2$, and this deviation increases with a decrease in $R_{\min }$.



Figure 11. Calculated $B_{\mathrm{Z}}(0)$ at the center of the wafer as a function of $R_{\min }$. Radius $a$ of the equivalent single-layer solenoid, which gives the same $B_{\mathrm{Z}}(0)$, is plotted as a function of $R_{\min }$. The mean radius of $R_{\max }$ and $R_{\min }, a^{\prime}$, is also plotted for comparison.

The $m$ wafer coils are supposed to be stacked to form a cylindrical unit of $2 R_{\text {wafer }}$ in diameter and $m \cdot t$ in height. The spiral coils on adjacent $S i$ wafers are supposed to be series-interconnected through two through-holes $[17,20]$ formed in the wafers by such a method illustrated in Figure $2 \mathrm{f}-\mathrm{h}$, which is frequently used in conventional multi-layer interconnection technology for 3D integration [17]. Thus, the series-interconnected stacked 
multiple wafer coils form a combined coil of inductance $L$. For simplicity in the calculation of inductance, $L$, these series-interconnected wafer coils were simulated to be a single-layer solenoid of the length $m \cdot t$ and of the equivalent radius $a$ given in Figure 11. If the number of turns of the coil in a wafer is $n, L$ can be expressed using the formula for the single-layer solenoid as follows:

$$
L=K \cdot \mu_{0} \cdot \mu_{r} \cdot \pi a^{2} \cdot \frac{(n \cdot m)^{2}}{m \cdot t}
$$

where $K$ is Nagaoka's coefficient, which is a function of $a /(m \cdot t), \mu_{\mathrm{r}}$ stands for relative magnetic permeability. In the case of $\mathrm{Si}$, it is reasonable to set $\mu_{\mathrm{r}}=1$. Figure 12 displays the calculated values of $L$ as a function of $R_{\min }$ in the case where the equivalent radius $a$ shown in Figure 11 was employed for the equivalent single-layer solenoid. The calculated values of $L$ in the case where the mean radius of the spiral coil $a^{\prime}=\left(R_{\max }+R_{\min }\right) / 2$ were employed for the equivalent single-layer solenoid, are also displayed for comparison. It was found that $L$ took the maximum value around $R_{\min }=13 \mathrm{~mm}$ when the equivalent radius, $a$, was employed, while $L$ showed no peak when the mean radius, $a^{\prime}$, was employed. The appearance of the maximum was caused by the variation in the product, $a \times n$, in Equation (5), where $a$ increased rapidly with $R_{\min }$ and $n$ decreased rapidly with $R_{\min }$. In contrast, the linear increase in $a^{\prime}$ with $R_{\min }$ did not cause a maximum in variation of the product $a^{\prime} \times n$ in Equation (5).

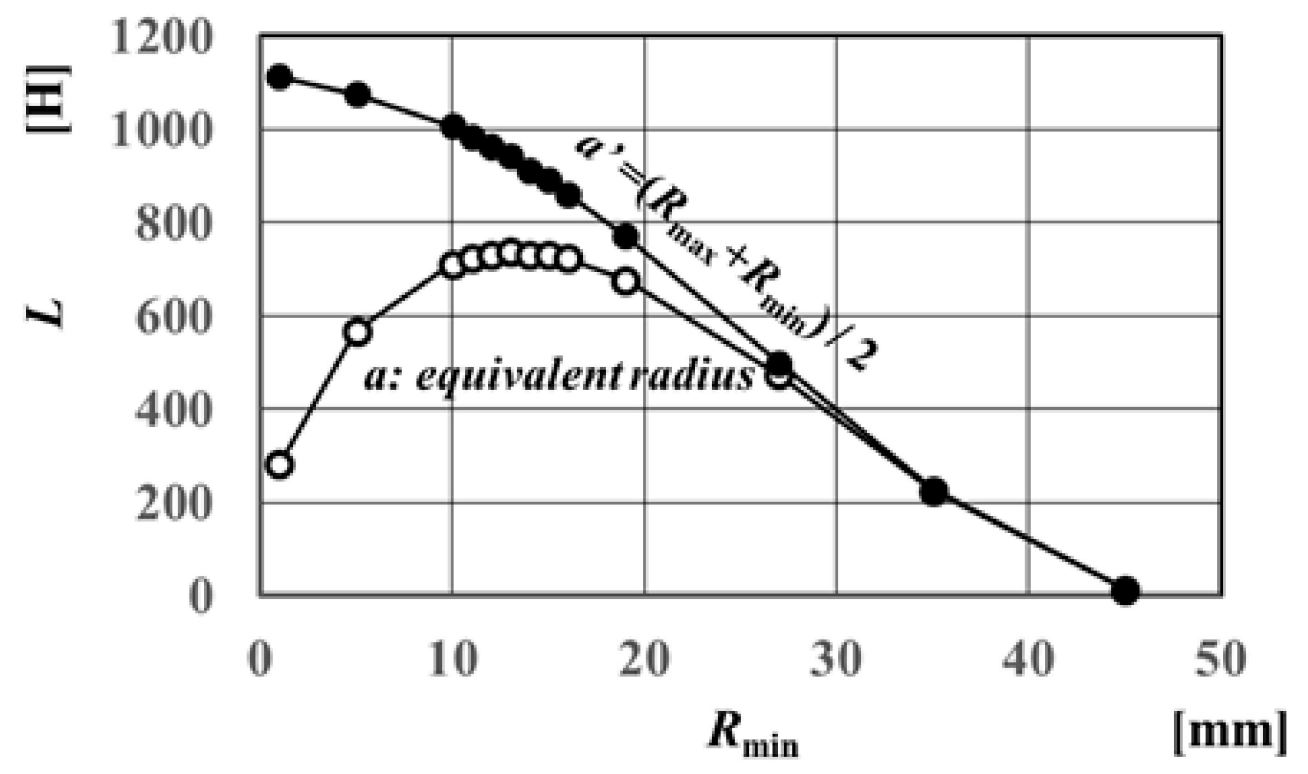

Figure 12. Inductance $L$ of the single-layer solenoid of the length $m \cdot t=30 \mathrm{~cm}$ as a function of $R_{\min }$ calculated employing the radius $a$ and $a^{\prime}$.

\subsection{Calculation of Electricity Storage Volume Density of the Wafer Coil Stack}

The electric energy $E$ stored in a solenoid of inductance $L$ can be expressed as:

$$
E=\frac{1}{2} L \cdot I^{2}
$$

Here, the superconducting current $I$, which flows in the coil at the critical current density $j_{\mathrm{c}}$ can be expressed as $I=d \cdot z \cdot j_{\mathrm{c}}$. Here, $w$ is calculated for the volume of cuboid including 4 stacks, as shown in Figure $1 c$ - that is, in the special case of $l=m \cdot t$. Because the volume of the cuboid in Figure $1 \mathrm{c}$ is $b \times b \times 2 h$, including the space for refrigerant, magnetic shields, heat insulation, and cryogenic refrigerator, $w=E \times 4 /(b \times b \times 2 h)$. Here, another volume of $b \times b \times h$ is supposed to be necessary for the cryogenic refrigerator in addition to the upper container of the volume of $b \times b \times h$ for 4 cylindrical wafer stack units. In this paper, estimations of $w$ were performed for the cases of $b=0.3 \mathrm{~m}$ and $t=0.5 \mathrm{~mm}$. 
The value $n$ can be expressed as $n=\left(R_{\max }+s-R_{\min }\right) /(d+s)$. Because $s / R_{\max } \ll 1$ and $s / R_{\min } \ll 1, n$ can be approximated as follows:

$$
n=\frac{R_{\max }-R_{\min }}{d+s}
$$

Then, (5) can be expressed as follows:

$$
L=K \cdot \mu_{0} \cdot \pi a^{2} \cdot \frac{\left(\frac{R_{\max }-R_{\min }}{d+s} \cdot m\right)^{2}}{m \cdot t}
$$

Then, $w$ can be expressed as follows:

$$
w=\frac{\frac{1}{2} K \cdot \mu_{0} \cdot \pi a^{2} \cdot \frac{\left(\frac{R_{\max }-R_{\min }}{d+s} \cdot m\right)^{2}}{m \cdot t} \cdot\left(d \cdot z \cdot j_{c}\right)^{2} \times 4}{b \times b \times 2 h}=\frac{2 K \cdot \mu_{0} \cdot \pi a^{2} \cdot R_{\max }{ }^{2}\left(1-\frac{R_{\min }}{R_{\max }}\right)^{2} \cdot\left(\frac{1}{1+\frac{s}{d}}\right)^{2} \cdot\left(z \cdot j_{c}\right)^{2}}{b \times b \times 2 h \cdot t / m}
$$

Because trenches cannot be formed on the peripheral ring area of the Si wafer with a width of at least $3 \mathrm{~mm}$ for the handling of the wafer in the series of processes, $R_{\max }$ can be set in a limited range and is essentially constant. Therefore, $w$ increases with a decreasing $R_{\min }$. Equation (9) also teaches us that $w$ increases with a decreasing $(s / d)$ and with increasing $z$. Because $h=m \cdot t$ in the special case drawn in Figure 1c, the denominator of Equation (9) does not contain the number of Si wafer coils, $m$. However, an increase in $m$ can contribute an increase in $w$, because $K$ increases with $m \cdot t / a$. A decrease in $a$ with a decrease in $R_{\min }$, as shown in Figure 11, can also contribute to an increase in $w$ via an increase in $K$. However, $w$ increases with $a$ because Equation (9) contains $a^{2}$ in the numerator. Therefore, $w$ can increase with an increasing $R_{\min }$. Thus, it is not straightforward to know the actual variation in $w$.

\subsection{Electromagnetic Hoop Stress}

Expressing free electron density as $\rho \mathrm{m}^{-3}$ and elementary charge $1.60 \times 10^{-7} \mathrm{C}$ as $e$, the charge $q \mathrm{C}$ in the superconducting film per length $\mathrm{d} x$ along the trench can be expressed as $q=e \cdot \rho \cdot d \cdot z \cdot d x$. Expressing the electron velocity as $v, I$ is expressed as $I=e \cdot \rho \cdot d \cdot z \cdot v$. The Lorenz force $F$ imposed on the current $I$ can be expressed as $F=q \cdot v \cdot B=I \cdot \mathrm{d} x \cdot \Sigma B_{\mathrm{Z}}(\mathrm{r})$. Because the corresponding surface area on the trench wall is $z \cdot \mathrm{d} x$, the hoop stress $S$ in the unit of $\mathrm{GPa}$ on the trench wall can be expressed as follows:

$$
S=\frac{I \cdot d x \cdot \sum B_{z}(r)}{z \cdot d x} \times 10^{-9}
$$

Because $S$ is normal to $B$ and $I, S$ gives the hoop stress, as shown in Figure 8a. When $\Sigma B_{\mathrm{z}}(\mathrm{r})$ takes the maximum value $\Sigma B_{\max }$ at the innermost trench, $S$ also takes the maximum value, $S_{\max }$. Figure 13 displays the variations in the electricity storage volume density, $w$, $S_{\max }$, and $\Sigma B_{\max }$, as a function of $R_{\min }$. 


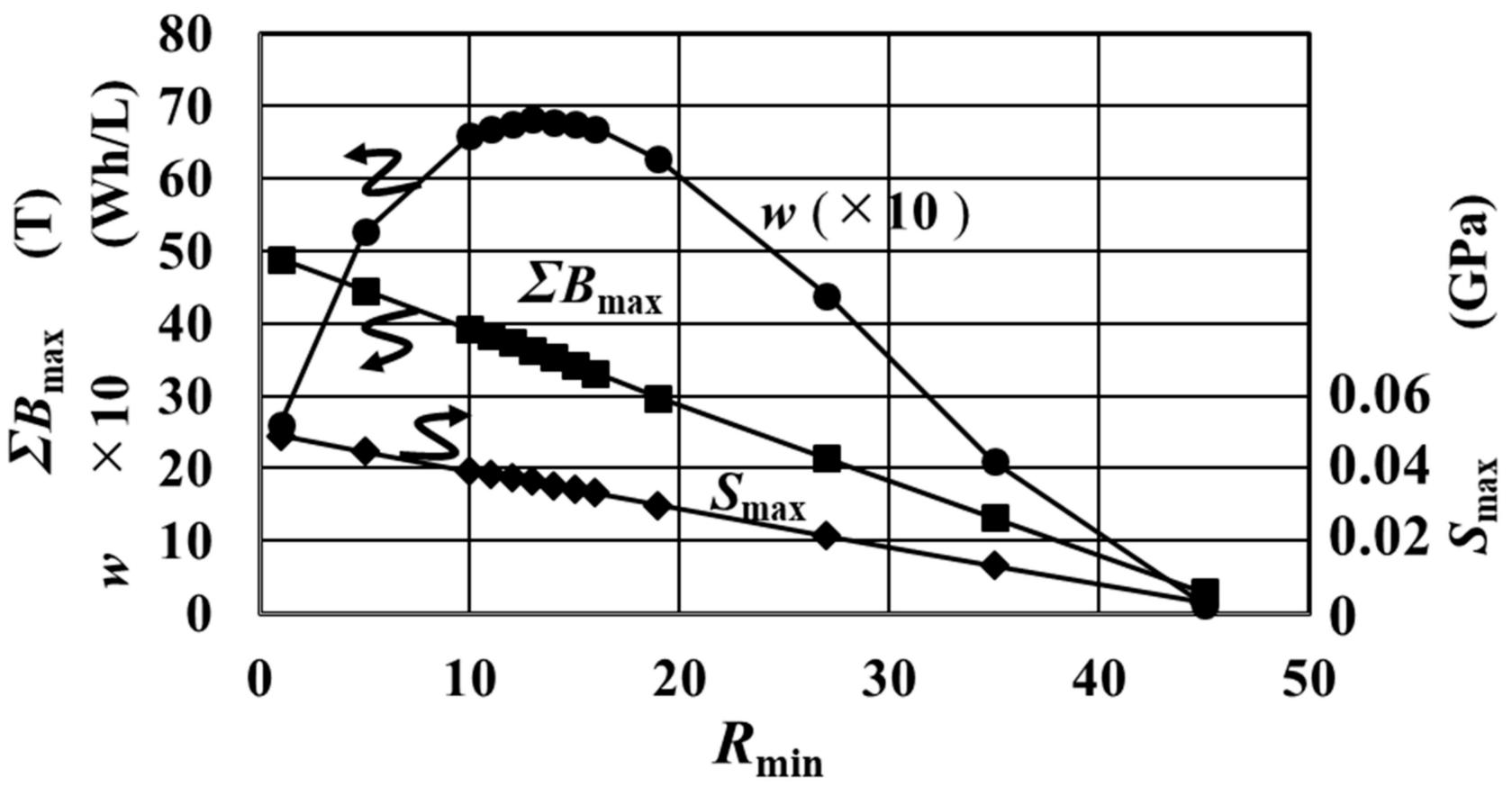

Figure 13. Variations in the electricity storage volume density $w, S_{\max }$, and $\Sigma B_{\max }$ as a function of $R_{\min }$ based on the magnetic flux density independent $j_{\mathrm{c}}=2 \times 10^{4} \mathrm{~A} / \mathrm{mm}^{2}$.

\subsection{Consideration of Magnertic Flux Density Dependent $j_{c}$ in the Calculation}

The results displayed in Figure 13 were calculated using the magnetic flux density independent $j_{\mathrm{c}}$ of $2 \times 10^{4} \mathrm{~A} / \mathrm{mm}^{2}$. In the next step, the magnetic flux density dependent $j_{c}$ shown in curves (b) and (c) in Figure 4 is taken into consideration. This is essentially an iteration process in which the $\Sigma_{\max }$ decided by the initially set value $j_{\mathrm{c} 0}$ reduces $j_{\mathrm{c} 0}$ to $j_{\mathrm{c} 1}$, and then $j_{\mathrm{c} 1}$ decides the new value of $\Sigma B_{\max 1}$, which reduces $j_{\mathrm{c} 1}$ to $j_{\mathrm{c} 2}$, and so on. For the convenience of calculation, $j_{\mathrm{c}}$ was expressed as a function of $\Sigma B_{\max }$ in expression (11) based on curves (b) and (c) in Figure 4.

$$
j_{c}\left[A / \mathrm{mm}^{2}\right]=\frac{85,800}{\sum B_{\max }[T]+0.672}+12,900
$$

This iteration process finally gave converged values of $j_{\cos }$ and $\Sigma B_{\max \infty}$. Figure 14 displays the $w$ and $S_{\max }$ obtained with Equations (9) and (10) using $j_{\text {cos }}$ and $\Sigma B_{\max \infty}$ as functions of $R_{\min }$. It is found that $w$ and $S_{\max }$ were much reduced in comparison with Figure 13. The perpendicular dashed-dotted red line was drawn to show the value of $R_{\min }$ at which $\Sigma B_{\max }$ took the value of $20 \mathrm{~T}$. The red open circle indicates that the $\Sigma B_{\max }-R_{\min }$ curve crosses the line of $\Sigma B_{\max }=20 \mathrm{~T}$. The corresponding value of $R_{\min }$ was $24.7 \mathrm{~mm}$. If superconductivity is retained up to this $\Sigma B_{\max }: 20 \mathrm{~T}$, it is shown that the value of $w$ : 3.6 Wh/L is attained at $R_{\min }: 24.7 \mathrm{~mm}$ where $S_{\max }$ takes the value of $0.017 \mathrm{GPa}$. The perpendicular dashed-two dotted blue line was drawn at the value of $R_{\min }$ at which $w$ took the maximum value of $4.3 \mathrm{Wh} / \mathrm{L}$. The corresponding value of $R_{\min }$ was $15.3 \mathrm{~mm}$, where $\Sigma B_{\max }$ and $S_{\max }$ took the values of $26.7 \mathrm{~T}$ and $0.021 \mathrm{GPa}$, respectively. Therefore, it was shown that the value of $w, 4.3 \mathrm{Wh} / \mathrm{L}$ could be attained if the wafer coils are superconductive up to $\Sigma B_{\max }=26.7 \mathrm{~T}$. Here, it should be noted that the equivalent radius $a$ does not change with the reduced critical current $I_{\mathrm{c}}$ because $B_{\mathrm{Z}}(0)$ is also reduced in proportion to the reduced $I_{\mathrm{C}}=n \cdot d \cdot z \cdot j_{\mathrm{C}}$, and the ratio $I / B_{\mathrm{Z}}(0)$ does not change in Equation (4). 


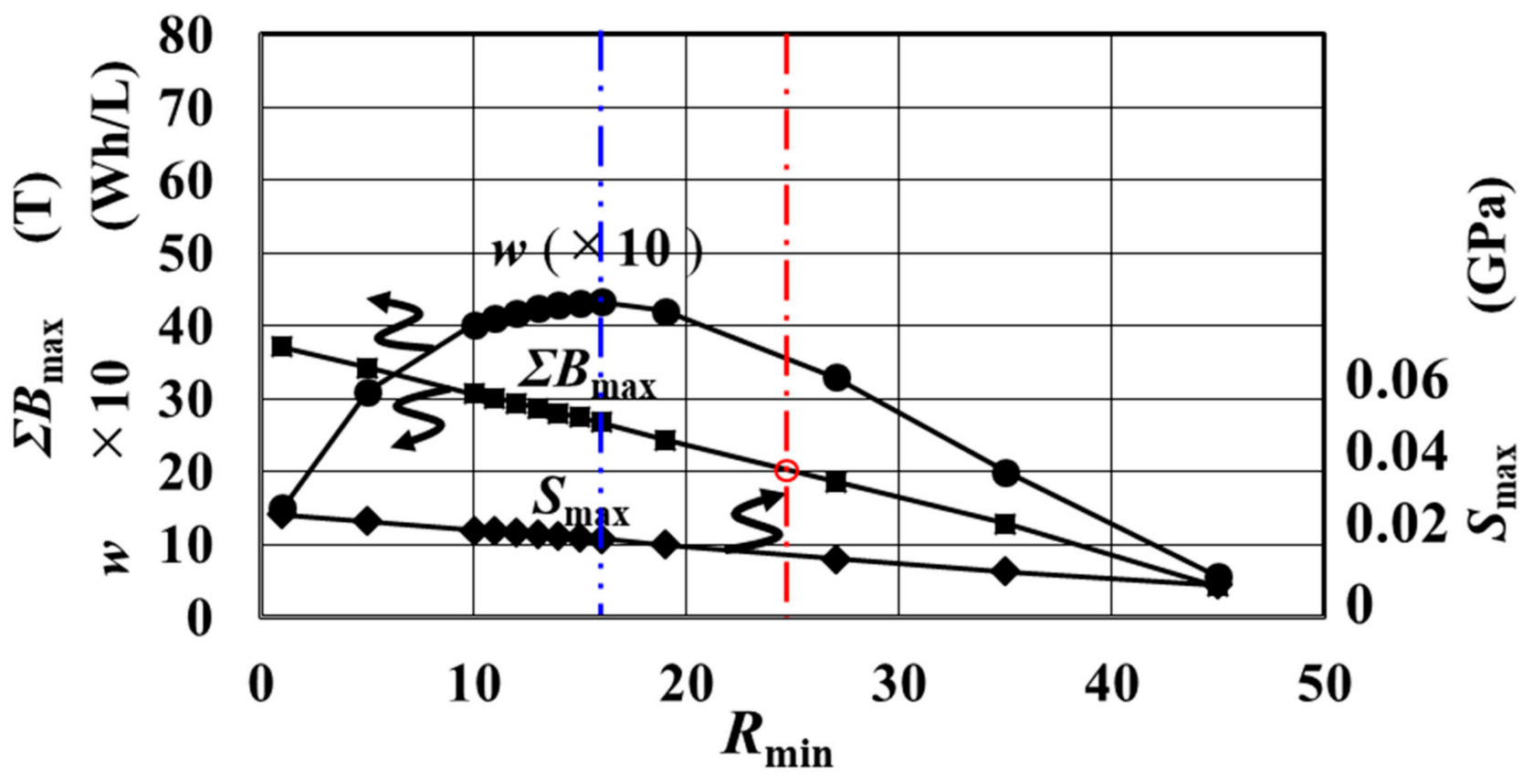

Figure 14. Variations in the electricity storage volume density $w, S_{\max }$, and $\Sigma B_{\max }$ as a function of $R_{\min }$ based on the magnetic flux density dependent $j_{\mathrm{c}}$ displayed as curves (b) and (c) in Figure 4.

\subsection{Effects of the Wafer Coil Design and the Hight of the Wafer Coil Stack on the Electricity Storage Volume Density}

The results shown in Figure 14 were obtained for the $\mathrm{Si}$ wafer with radius $R_{\text {wafer }}=50.8 \mathrm{~mm}$, the radius of the outer wall of the outermost trench $R_{\max }=47.8 \mathrm{~mm}$, and the thickness $t=0.5 \mathrm{~mm}$ in the case of the trench width $d=50 \mu \mathrm{m}$, the trench wall thickness $s=22 \mu \mathrm{m}$, the trench depth $z=30 \mu \mathrm{m}$, and the number of stacked Si wafers $m=600$. Here, the effects of changing $d, s, z$, and $m$ in practically viable ranges on $\Sigma B_{\max }, w$, and $S_{\max }$ were examined.

As mentioned above, Equation (9) teaches us that $w$ increases with decreasing $(s / d)$ and with increasing $z$. Because $w$ does not depend on the absolute values of $d$ and $s$ but depends on the ratio $s / d$, the examination of the different values of the sum $d+s$ is not necessary. Therefore, the cases of $d=70 \mu \mathrm{m}$ and $\mathrm{s}=2 \mu \mathrm{m}$ were examined at the same sum $d+s$ in the case of $d=50 \mu \mathrm{m}$ and $s=22 \mu \mathrm{m}$. As was elucidated in Figures $8 \mathrm{~b}$ and 9, the contributions of the stacked wafers at a distance more than $5 \mathrm{~cm}$ to $\Sigma B_{z}(r)$ are negligible. The distance of $5 \mathrm{~cm}$ in the stack corresponds to 100 wafer coils. Therefore, cases of $m=200$ in addition to cases of $\mathrm{m}=600$ were examined. Although the change in $m$ from 600 to 200 decreases $L$, as shown in Figure 15, and hence the amount of the magnetic energy storage for each cylindrical coil decreases, eight more cylindrical coil units can be installed in the cryogenic container of the volume $b \times b \times h$, as shown in Figure 16, which in turn increases $w$. Although $w$ increases with an increasing $z$, there is an experimental limitation in increasing $z$ because of the following reasons. The high-temperature heat treatment of the wafer coil in an oxygen atmosphere is indispensable for the crystallization and adjustment of oxygen deficiency $\delta$ in $\mathrm{YBa}_{2} \mathrm{Cu}_{3} \mathrm{O}_{7-\delta}$. This heat treatment induces the diffusion of $\mathrm{Si}$ into the Y123 layer, which degrades the superconducting property of Y123. To prevent this, some intermediate buffer layers are necessary between Si substrates and Y123 layers. The buffer layers are also necessary to mitigate lattice and thermal expansion coefficient mismatches between $\mathrm{Y} 123$ and Si. For these reasons, the sputter deposition of the yttria-stabilized zirconia layer succeeded by the sputter deposition of the $\mathrm{CeO}_{2}$ layer was used to form the intermediate buffer layers [34,35], and we also employed these buffer layers in our previous experimental study [27]. Because the throwing power of sputter deposition into the trench is limited, the large aspect ratio of the trench, $z / d$, cannot be 
employed. Therefore, cases of $z=100 \mu \mathrm{m}$ were examined as a realistic value in addition to cases of $z=30 \mu \mathrm{m}$. Figure 17 schematically summarizes the combinations of the wafer coil design parameters, $d, s, z$, examined here.

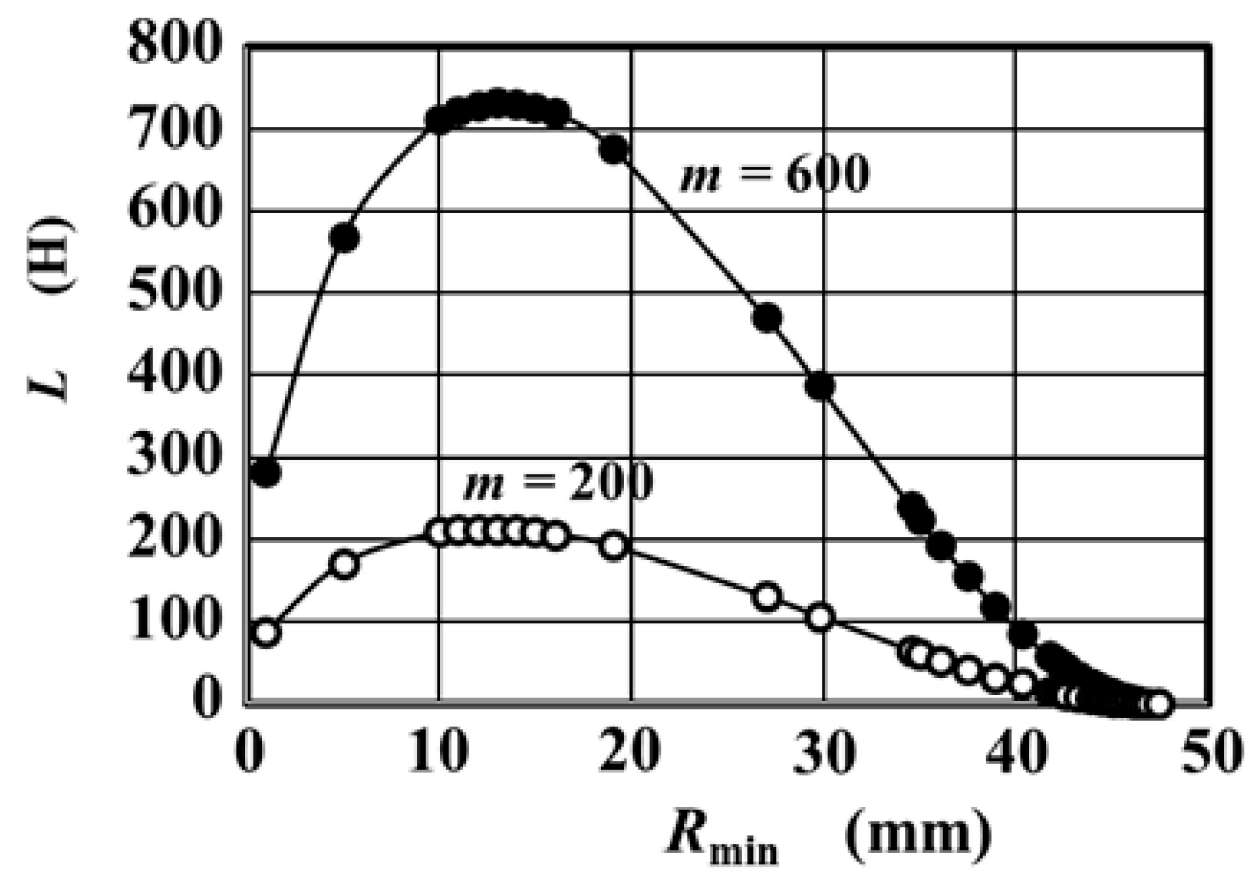

Figure 15. Dependence of $L$ on $R_{\min }$ for the cases of $m=600$ and $m=200$.

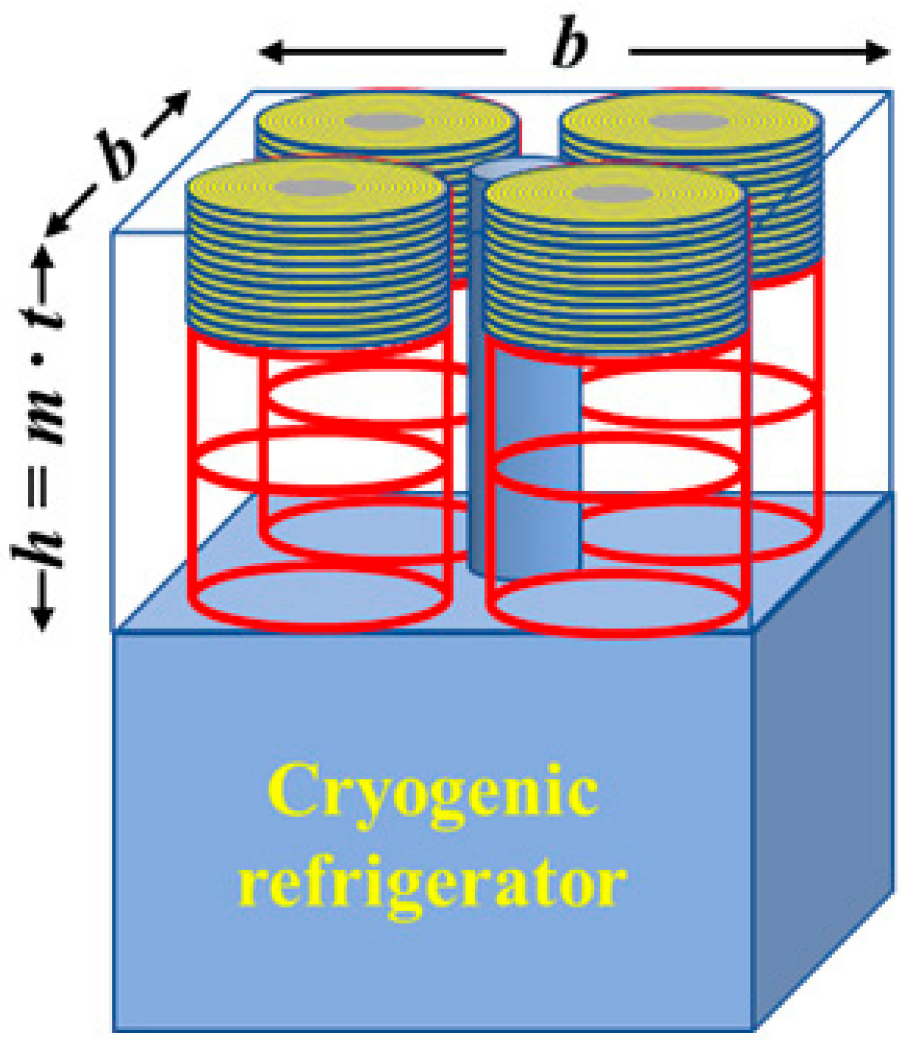

Figure 16. Decrease in $m$ from 600 to 200 and the installation of eight more wafer stacks in comparison with Figure 1c. 


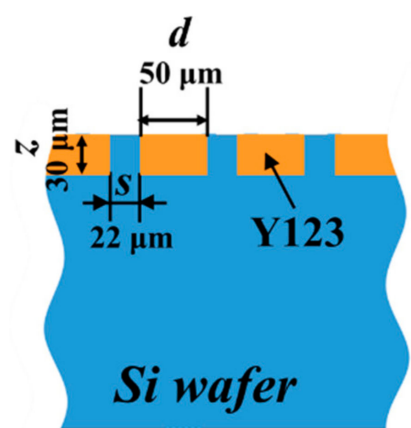

(a)

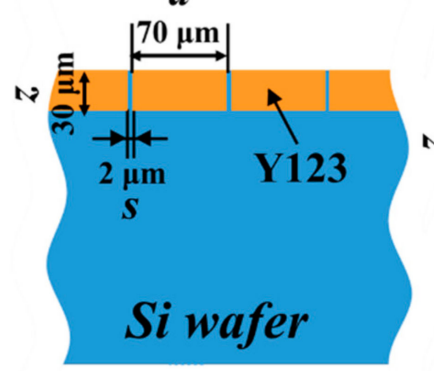

(c)

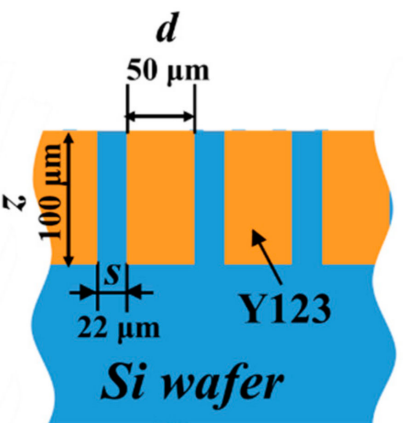

(b)

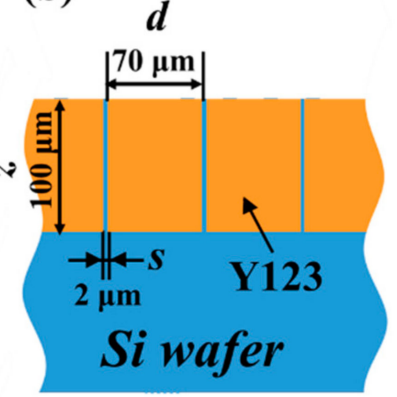

(d)

Figure 17. Schematic summary of the combinations of the wafer coil design parameters, $d, s, z$, examined. (a) $d=50 \mu \mathrm{m}, s=22 \mu \mathrm{m}, z=30 \mu \mathrm{m}$, (b) $d=50 \mu \mathrm{m}, s=22 \mu \mathrm{m}, z=100 \mu \mathrm{m}$, (c) $d=70 \mu \mathrm{m}$, $s=2 \mu \mathrm{m}, z=30 \mu \mathrm{m},(\mathbf{d}) d=70 \mu \mathrm{m}, s=2 \mu \mathrm{m}, z=100 \mu \mathrm{m}$.

2.8. Comparison of the Effects of the Wafer Coil Design and the Height of the Wafer Coil Stack under Two Different Magnetic Flux Density-Dependent $j_{c}$

Like Equation (11) for curves (b) and (c) in Figure $4, j_{\mathrm{c}}$ was also expressed as a function of $\Sigma B_{\max }$ based on curve (a) in the following expression (12):

$$
j_{c}\left[A / m m^{2}\right]=\frac{59,400}{\sum B_{\max }[T]+1.75}+9630
$$

Iteration processes using Equation (11) or Equation (12) gave converging values of $j_{\mathrm{c} \infty}$. and $\Sigma B_{\max \infty}$ as a function of $R_{\min }$. Then, $j_{\mathrm{c} \infty}$. and $\Sigma B_{\max \infty}$ gave $w$ and $S_{\max }$ as a function of $R_{\min }$.

\section{Results}

The results of the calculations of $\Sigma B_{\max }, w$, and $S_{\max }$ as a function of $R_{\min }$ for two different value settings of $m, d, s$, and $z$ shown in Figures 16 and 17 are displayed in the same manner as in Figure 14; in Figures S1-S4 in the case of the magnetic flux densitydependent $j_{\mathrm{c}}$ of curves (b) and (c) in Figure 4, which is simulated by Equation (11); and in Figures S5-S8 in the case of the magnetic flux density-dependent $j_{\mathrm{C}}$ of curve (a) in Figure 4 , which is simulated by Equation (12), respectively. The values of $w, R_{\min }, S_{\max }$, and $\Sigma B_{\max }$ indicated by the perpendicular dashed-dotted red lines and the perpendicular dashed-two dotted blue lines in Figures S1-S8 are summarized in Table 1. In Table 1, $w$ @20T and $w$ peak stand for $w$ at $\Sigma B_{\max }=20 \mathrm{~T}$ and the peak values of $w$, respectively. In the case of the $\Sigma B_{\mathrm{Z}}(r)$ dependence of $j_{\mathrm{c}}$ expressed by Equation (11) (curves (b) and (c) in Figure 4), $w_{@ 20 \mathrm{~T}}$ took values between 3.5 and $4.5 \mathrm{Wh} / \mathrm{L}$ in the range of $R_{\min } 21.5-31.4 \mathrm{~mm}$ when $z=30 \mu \mathrm{m}$, while $w_{@ 20 \mathrm{~T}}$ took values between 5 and $6.8 \mathrm{Wh} / \mathrm{L}$ in the range of $R_{\min } 40-43.5 \mathrm{~mm}$ when $z=100 \mu \mathrm{m}$. An increase in $d$ from 50 to $70 \mu \mathrm{m}$ also caused an increase in $w_{@ 20 \mathrm{~T}}$ and $R_{\min }$. The difference in $m$ between that illustrated in Figure $1 c$ and in Figure 16 did not cause simple trends of increase nor decrease in $w_{@ 20 \mathrm{~T}}$ but caused a slight decrease in $R_{\min }$ with decreasing $m$. 
Table 1. Calculated values of $w, R_{\min }, S_{\max }$, and $\Sigma B_{\max }$ for the different wafer coil design parameters, $d, s, z$, and the number of the stacked wafer coils, $m$, under the different $\Sigma B_{\max }$ dependences of $j_{\mathrm{c}}$.

\begin{tabular}{|c|c|c|c|c|c|c|c|c|c|c|c|c|c|}
\hline Fig. & $j_{\mathrm{c}}-B$ Curve & $\underset{(\mu \mathrm{m})}{d}$ & $\begin{array}{c}s \\
(\mu \mathrm{m})\end{array}$ & $\begin{array}{c}z \\
(\mu \mathrm{m})\end{array}$ & $m$ & $\begin{array}{l}w_{@ 20 T} \\
(\mathrm{Wh} / \mathrm{L})\end{array}$ & $\begin{array}{l}R_{\min } \\
(\mathrm{mm})\end{array}$ & $\begin{array}{l}S_{\max } \\
(\mathrm{GPa})\end{array}$ & $\begin{array}{c}B_{\max } \\
\text { (T) }\end{array}$ & $\begin{array}{c}w_{\text {peak }} \\
(\mathrm{Wh} / \mathrm{L})\end{array}$ & $\begin{array}{l}R_{\min } \\
(\mathrm{mm})\end{array}$ & $\begin{array}{l}S_{\max } \\
(\mathrm{GPa})\end{array}$ & $\underset{(\mathrm{T})}{\sum \boldsymbol{B}_{\max }}$ \\
\hline Figure S1a & \multirow{8}{*}{$\begin{array}{c}\text { (b), (c) in Figure } 4 \\
\text { Equation (11) }\end{array}$} & \multirow{4}{*}{50} & \multirow{4}{*}{22} & 30 & \multirow[b]{2}{*}{600} & 3.6 & 24.7 & 0.017 & \multirow{8}{*}{20} & 4.3 & 15.3 & 0.021 & 26.7 \\
\hline Figure S1b & & & & 100 & & 5 & 40.6 & 0.020 & & 36.5 & 13.7 & 0.057 & 81.4 \\
\hline Figure S2a & & & & 30 & & 3.5 & 21.5 & 0.017 & & 3.9 & 14.8 & 0.020 & 24.0 \\
\hline Figure S2b & & & & 100 & 200 & 5.8 & 40.0 & 0.017 & & 33.8 & 13.5 & 0.052 & 74.2 \\
\hline Figure S3a & & \multirow{4}{*}{70} & \multirow{4}{*}{2} & 30 & \multirow[b]{2}{*}{600} & 4.3 & 31.4 & 0.024 & & 7.6 & 15.0 & 0.038 & 36.0 \\
\hline Figure S3b & & & & 100 & & 6.8 & 43.5 & 0.020 & & 69.4 & 13.7 & 0.105 & 110 \\
\hline Figure S4a & & & & 30 & & 4.5 & 29.3 & 0.024 & & 6.9 & 14.6 & 0.035 & 32.0 \\
\hline Figure S4b & & & & 100 & 200 & 6 & 42.5 & 0.020 & & 60.0 & 13.9 & 0.094 & 99.0 \\
\hline Figure S5a & \multirow{8}{*}{$\begin{array}{l}\text { (a) in Figure } 4 \\
\text { Equation (12) }\end{array}$} & \multirow{4}{*}{50} & \multirow{4}{*}{22} & 30 & \multirow[b]{2}{*}{600} & 5.1 & 36.9 & 0.038 & \multirow{8}{*}{20} & 9.8 & 19.0 & 0.047 & 37.0 \\
\hline Figure S5b & & & & 100 & & 6.1 & 44.5 & 0.038 & & 48.2 & 17.5 & 0.070 & 86.1 \\
\hline Figure S6a & & & & 30 & & 5.6 & 34.9 & 0.038 & & 9.3 & 19.4 & 0.045 & 33.8 \\
\hline Figure S6b & & & & 100 & 200 & 6.7 & 43.7 & 0.038 & & 44.9 & 17.4 & 0.066 & 77.8 \\
\hline Figure S7a & & \multirow{4}{*}{70} & \multirow{4}{*}{2} & 30 & \multirow[b]{2}{*}{600} & 5.1 & 40.3 & 0.052 & & 14.9 & 18.8 & 0.072 & 42.6 \\
\hline Figure S7b & & & & 100 & & 5.6 & 45.8 & 0.052 & & 80.0 & 17.5 & 0.114 & 110 \\
\hline Figure S8a & & & & 30 & \multirow[b]{2}{*}{200} & 5.1 & 39.8 & 0.052 & & 14.1 & 19.1 & 0.068 & 41.6 \\
\hline Figure S8b & & & & 100 & & 6.4 & 45.4 & 0.053 & & 73.6 & 16.6 & 0.011 & 102 \\
\hline
\end{tabular}

In the case of the $\Sigma B_{\mathrm{Z}}(r)$ dependence of $j_{\mathrm{c}}$ expressed by Equation (12) (curve (a) in Figure 4$), w_{@ 20 \mathrm{~T}}$ took values between 5.1 and $5.6 \mathrm{Wh} / \mathrm{L}$ in the range of $R_{\min } 34.9-40.3 \mathrm{~mm}$ when $z=30 \mu \mathrm{m}$, while $w_{@ 20 \mathrm{~T}}$ took the values between 6.1 and $6.7 \mathrm{Wh} / \mathrm{L}$ in the range of $R_{\min } 43.7-45.8 \mathrm{~mm}$ when $z=100 \mu \mathrm{m}$. An increase in $d$ from 50 to $70 \mu \mathrm{m}$ caused an increase in $R_{\min }$ but did not cause an increase in $w_{@ 20 \mathrm{~T}}$. A decrease in $m$ from 600 to 200 caused a slight increase in $w_{20}$ and slight decrease in $R_{\min }$.

In the case of the $\Sigma B_{z}(r)$ dependence of $j_{c}$ expressed by Equation (11) (curves (b) and (c) in Figure 4$), w_{\text {peak }}$ took values between 3.9 and $7.6 \mathrm{Wh} / \mathrm{L}$ in the range of $R_{\min } 13.5-13.9 \mathrm{~mm}$ when $z=30 \mu \mathrm{m}$, while $w_{\text {peak }}$ took values between 33.8 and $69.4 \mathrm{Wh} / \mathrm{L}$ in the range of $R_{\min } 40-43.5 \mathrm{~mm}$ when $z=100 \mu \mathrm{m}$. An increase in $d$ from 50 to $70 \mu \mathrm{m}$ also caused an increase in $w_{\text {peak. }}$. A decrease in $m$ from 600 to 200 caused a decrease in $w_{\text {peak }}$.

In the case of the $\Sigma B_{\mathrm{Z}}(r)$ dependence of $j_{\mathrm{c}}$ expressed by Equation (12) (curve (a) in Figure 4$), w_{\text {peak }}$ took values between 9.3 and $14.9 \mathrm{Wh} / \mathrm{L}$ in the range of $R_{\min } 18.8-19.4 \mathrm{~mm}$ when $z=30 \mu \mathrm{m}$, while $w_{\text {peak }}$ took values between 48.2 and $80 \mathrm{Wh} / \mathrm{L}$ in the range of $R_{\min } 16.6-17.5 \mathrm{~mm}$ when $z=100 \mu \mathrm{m}$. An increase in $d$ from 50 to $70 \mu \mathrm{m}$ caused an increase in $w_{\text {peak }}$ but did not cause an increase in $R_{\min }$. A decrease in $m$ from 600 to 200 caused a decrease in $w_{\text {peak }}$.

Although the values of $w_{\text {peak }}$ often exceeded $10 \mathrm{Wh} / \mathrm{L}$ and sometimes approached $100 \mathrm{Wh} / \mathrm{L}$ when $z=100 \mu \mathrm{m}$ or $d=70 \mu \mathrm{m}$, the corresponding values of $\Sigma B_{\max }$ often exceeded $50 \mathrm{~T}$ and $100 \mathrm{~T}$ in some cases. These large values of $\Sigma B_{\max }$ may exceed the critical magnetic flux density, although there are no signs of the appearance of critical magnetic flux density within the range shown in Figure 4. Table 2 lists the values of $w$ taken from Figures S1-S8 at the $\Sigma B_{\max }$ of every $10 \mathrm{~T}$ increment up to $w_{\text {peak }}$. 


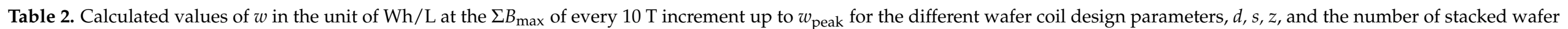
coils, $m$, under the different $\Sigma B_{\max }$ dependences of $j_{\mathrm{c}}$. Expressions such as $w_{@ 20 \mathrm{~T}}$ indicate $w$ when the $\Sigma B_{\max }$ is $20 \mathrm{~T}$.

\begin{tabular}{|c|c|c|c|c|c|c|c|c|c|c|c|c|c|c|c|}
\hline Fig. & $\begin{array}{l}j_{\mathrm{c}}-B \\
\text { Curve }\end{array}$ & $d(\mu \mathrm{m})$ & $s(\mu \mathrm{m})$ & $z(\mu \mathrm{m})$ & $\mathbf{m}$ & $\begin{array}{l}w_{@ 20 \mathrm{~T}} \\
(\mathrm{Wh} / \mathrm{L})\end{array}$ & $\begin{array}{l}w_{@ 30 T} \\
(\mathrm{Wh} / \mathrm{L})\end{array}$ & $\begin{array}{l}w_{@ 40 \mathrm{~T}} \\
(\mathrm{Wh} / \mathrm{L})\end{array}$ & $\begin{array}{l}w_{@ 50 T} \\
(\mathrm{Wh} / \mathrm{L})\end{array}$ & $\begin{array}{l}w_{@ 66 \mathrm{~T}} \\
(\mathrm{Wh} / \mathrm{L})\end{array}$ & $\begin{array}{l}w_{@ 70 \mathrm{~T}} \\
(\mathrm{Wh} / \mathrm{L})\end{array}$ & $\begin{array}{l}w_{\Theta 80 \mathrm{~T}} \\
(\mathrm{Wh} / \mathrm{L})\end{array}$ & $\begin{array}{l}w_{@ 90 T} \\
(\mathbf{W h} / \mathrm{L})\end{array}$ & $\begin{array}{l}w_{@ 100 T} \\
(\mathbf{W h} / \mathrm{L})\end{array}$ & $\begin{array}{c}w_{\text {peak }} @ \sum B_{\max } \\
(\mathrm{Wh} / \mathrm{L})\end{array}$ \\
\hline Figure S1a & \multirow{6}{*}{$\begin{array}{c}\text { (b,c)in Figure } 4 \\
\text { Equation (11) }\end{array}$} & \multirow{3}{*}{50} & \multirow{3}{*}{22} & 30 & \multirow{2}{*}{600} & 3.6 & & & & & & & & & $4.3 @ 26.7 \mathrm{~T}$ \\
\hline Figure S1b & & & & 100 & & 5 & 11.3 & 18.4 & 25 & 30 & 34.8 & & & & 36.5@81.4T \\
\hline Figure S2b & & & & 100 & 200 & 5.75 & 12 & 18.5 & 25 & & & & & & $33.8 @ 74.2 \mathrm{~T}$ \\
\hline Figure S3a & & \multirow{3}{*}{70} & \multirow{3}{*}{2} & 30 & \multirow{2}{*}{600} & 4.3 & 6.9 & & & & & & & & 7.6@36T \\
\hline Figure S3b & & & & 100 & & 6.8 & 8.6 & 19.8 & 27.8 & 39 & 47.8 & 55.6 & 62.5 & 67.4 & 69.4@110T \\
\hline Figure S4b & & & & 100 & 200 & 6 & 13.4 & 20 & 29.5 & 37.4 & 47.6 & 53.8 & & & $60.0 @ 99 \mathrm{~T}$ \\
\hline Figure S5a & \multirow{6}{*}{$\begin{array}{l}\text { (a) in Figure } 4 \\
\text { Equation (12) }\end{array}$} & \multirow{4}{*}{50} & \multirow{4}{*}{22} & 30 & \multirow{2}{*}{600} & 5.1 & & & & & & & & & $9.8 @ 37 \mathrm{~T}$ \\
\hline Figure S5b & & & & 100 & & 6.1 & 14 & 20 & 30.2 & 39.6 & 43 & & & & 48.2@86.1T \\
\hline Figure S6a & & & & 30 & \multirow{2}{*}{200} & 5.6 & & & & & & & & & 9.3@33.8T \\
\hline Figure S6b & & & & 100 & & 6.7 & 14.4 & 22.2 & 30.6 & 38.6 & 42.9 & & & & 44.9@77.8T \\
\hline Figure S7a & & \multirow[b]{2}{*}{70} & \multirow[b]{2}{*}{2} & 30 & \multirow{2}{*}{600} & 5.1 & 9.8 & 14 & & & & & & & $14.9 @ 42.6 \mathrm{~T}$ \\
\hline Figure S7b & & & & 100 & & 5.6 & 10 & 22.3 & 31 & 43.6 & 52.7 & 62.6 & 69.5 & & $80.0 @ 110 \mathrm{~T}$ \\
\hline
\end{tabular}


Table 2 shows that the values of $w_{@ 20 \mathrm{~T}}$ at $\Sigma B_{\max }=20 \mathrm{~T}$ are lower than $10 \mathrm{Wh} / \mathrm{L}$ in all the different wafer coil design parameters, $d, s, z$, and the number of stacked wafer coils $m$ under the different $\Sigma B_{\max }$ dependences of $j_{\mathrm{c}}$. In the cases of $z=100 \mu \mathrm{m}$ and in some cases of $d=70 \mu \mathrm{m}$, the values of $w$ at $\Sigma B_{\max }$ are higher than $20 \mathrm{~T}$ and can be larger than $10 \mathrm{Wh} / \mathrm{L}$, or more with an increase in $\Sigma B_{\max }$. It is found that this trend is more enhanced in the case of the $\Sigma B_{z}(r)$ dependence of $j_{c}$ expressed by Equation (12) (curves (a) in Figure 4) than in the case of the $\Sigma B_{\mathrm{Z}}(r)$ dependence of $j_{\mathrm{c}}$ expressed by Equation (11) (curves (b) and (c) in Figure 4). In all conditions, the values of $S_{\max }$ were found to take values well below $1 / 3$ of the mechanical strength limit of the $\mathrm{Si}$ wafer $\sigma_{\mathrm{Si}}: 4 \mathrm{GPa}$.

\section{Discussion}

\subsection{Possibility of SMES to Rank with or Surpass Capacitors in Electricity Storage Volume Density}

The dependence of $j_{c}$ of Y123 thin films on the magnetic flux density is sensitively affected by many experimental factors such as the methods of deposition, deposition conditions, substrates, buffer layers, etc. The data displayed in Figure 4 are the dependences of $j_{c}$ on magnetic flux density in parallel with the c-axis of preferentially c-axis-oriented Y123 thin films. Y123 thin films sometimes grow in mixed orientations of the c-axis and aaxis [25,27]. Even if c-axis-oriented Y123 is successfully grown on buffered Si substrate [26], the c-axis of the Y123 thin film grown on the bottom of the trench and the c-axis of the Y123 thin film grown on the trench wall cannot be in parallel with the same magnetic flux simultaneously. Therefore, there remains many challenges in material science and engineering to attain depositions of high-quality Y123 thin films with a sufficient magnetic flux density dependence of $j_{c}$ in the trench. However, the existence of the experimental evidence shown in Figure 4 encourages us to develop a compact SMES with the new concept presented in this work, which can rank with or surpass the capacitors on the market in terms of electricity storage volume density. The results summarized in Table 2 teach us that $w$ can rank with the commercially available capacitors, including EDLC, if superconductivity is retained up to the magnetic flux density $\Sigma B_{\max }$ of $20 \mathrm{~T}$. The results also teach us that $w$ can surpass capacitors including EDLC if superconductivity is retained up to a $\Sigma B_{\max }$ of $30 \mathrm{~T}$. According to the experimental evidence of curve (b) and (c) shown in Figure 4, superconductivity is retained up to $\Sigma B_{\max }=18 \mathrm{~T}$. It will be duly considered that superconductivity is retained up to $20 \mathrm{~T}$. According to curve (a), superconductivity is retained up to a $\Sigma B_{\max }$ of $31 \mathrm{~T}$. Moreover, there is a possibility that superconductivity is retained beyond $40 \mathrm{~T}$, because there is no indication of a rapid decrease in $j_{\mathrm{c}}$ due to the accession of the critical magnetic flux density $B_{\mathrm{c}}$. In this case, $w$ may rank with commercially available hybrid energy storage capacitors [4]. According to the results summarized in Table 2 for the different trench design parameters illustrated in Figure 17, there remains possibilities to attain a greater $w$ by using deeper and wider trenches within the experimentally feasible ranges.

\subsection{Improvement for Lower Cost and Compatibility for Mass Production}

According to Table 1, a decrease in $m$ from 600 to 200 accompanied by an increase in the number of wafer stacks from 4 to 12 as illustrated in Figure 16 caused no significant effect on $w$. This is good news because the process to stack 200 wafer coils is much easier than the process to stack 600 wafer coils from the viewpoint of a reduction in yield loss and process cost. The semiconductor microfabrication processes used here for the fabrication of the units of stacked wafer coils are compatible in nature with unattended automatic mass production and have the possibility to yield a significant cost reduction, which has been attained in the production of solar panels in which several tens of Si wafers are installed in one product (panel), just like the compact SMES proposed here. However, it is a great challenge to reduce the process cost of the repeated reactive etching process for trench formation and multiple wafer-bonding processes. In April 2018, the present authors noticed the announcement of the commercialization of a compact analyzer gas chromatograph, which is more than $30 \%$ smaller and gives $50 \%$ less analysis time compared 
with the conventional ones equipped with capillary columns [36]. The main part of this product is made of spiral trenches formed in a stainless steel substrate and stacked with another stainless steel plate as a lid in place of the capillary columns.

The development of this product started with the microfabrication of spiral trenches on Si wafers [37], just like the present study. According to the result summarized in Table 1, the values of the maximum hoop stress $S_{\max }$ induced on the trench wall were less than 0.2 $\mathrm{GPa}$, which was well below the $1 / 3$ mechanical strength limit of the $\mathrm{Si}$ wafer $\sigma_{\mathrm{Si}}, 4 \mathrm{GPa}$. This fact indicates that other substrate materials such as typical engineering ceramics or metals can be used in place of a Si wafer if an imprinting process or molding process for spiral trenches is feasible. Therefore, the compact SMES in the proposed concept may follow in the same footsteps of development of the compact analyzer gas chromatograph mentioned above. This possibility of the employment of substrate materials other than $\mathrm{Si}$ may also open the door to other applications of the present wafer coils besides SMES - such as, for example, magnetic resonators for wireless power transfer systems with a longer transfer range utilizing the high-quality factor of superconducting coils [38].

\subsection{Applications of the Compact SMES Which Ranks with or Surpass the Commercially Available Capacitors}

A SMES has been developed for a power system control such as load fluctuation compensation and power system stabilization [6-10,39]. However, those infrastructural applications have been proposed for usage in the space of a loosely restricted volume condition based on the electricity storage volume density of the sub-Wh/L level of conventional SMESs. Therefore, the benefit of the compact SMES proposed in the present study is limited. Of course, the compact SMES of the proposed concept may be distributed widely in the market by mass production and may contribute to these conventional applications at a lower cost and in a much reduced volume. Here, let us focus one of the outstanding features of the SMES: its rapid high-power delivery of around MW/msec. This feature makes the SMES suitable for pulsed power load operation [40]. An extreme example of a pulsed power load is a lightning strike. Controlling lightning strikes to prevent them from causing damage to various infrastructures and facilities is a serious task for telecom companies. Recently, there was an announcement that Nippon Telegraph and Telephone Corporation (NTT) started a study of technology to capture and guide lightning strikes [41]. In the project of NTT, it is planned that drones flying near the thunder clouds guide lightning electricity with the attached conductive wire to the ground in place of the kite used by Benjamin Franklin. In the project, it is also considered to guide the lightning electricity with the conductive wire to an electricity storage facility on board of a car. Reportedly, there is a wide variety of the properties of lightnings with their current from $1 \mathrm{kA}$ to $200 \mathrm{kA}$, with their voltage from $2 \mathrm{MV}$ to $200 \mathrm{MV}$, with their pulse width from sub-msec to $1 \mathrm{sec}$, and power from $10 \mathrm{kWh}$ to $500 \mathrm{kWh}$. If we take the values of the most frequent occurrences, the electricity pulse of lightning is typically $25 \mathrm{kA}$, the voltage is typically $29 \mathrm{MV}$, the pulse width is typically $1 \mathrm{msec}$, and the power is typically $200 \mathrm{kWh}$. The pulse width is too narrow to charge rechargeable batteries. The corresponding voltage of $29 \mathrm{MV}$ may be too high for conventional capacitors to be charged. As for SMES, a feasibility study for energy regeneration on-board ground vehicles has been reported, although the proposed system was still as large as about $1 \mathrm{~m}$ in diameter and heavy, with bulky reinforced structures against the hoop stress [16]. The total electricity of $200 \mathrm{kWh}$ of one lightning requests the volume of $400 \mathrm{~m}^{3}$ if the typical conventional electricity storage volume density $w$ of $0.5 \mathrm{Wh} / \mathrm{L}$ is employed. This is too large to install on-board a car. However, the compact SMES of the proposed concept in this work requests a volume of $40 \mathrm{~m}^{3}$ if $w$ is $5 \mathrm{Wh} / \mathrm{L}$. This compact SMES can be installed on-board a standard 4-ton truck. Although it goes without saying that SMES also requires improved insulation barriers and more detailed engineering efforts, this topic is mentioned here to elucidate the merit of the compact SMES of the proposed concept. 


\section{Conclusions}

SMESs store electricity as the energy of a magnetic field in contrast to capacitors, which store electricity as the energy of an electric field. Because SMESs and capacitors store electricity based on purely physical phenomena, their abilities of rapid charge and discharge are specific to them in contrast to rechargeable batteries based on an electrochemical phenomenon. However, SMESs are heavy electric machinery products of a relatively high cost, which is caused by their made-to-order production, while varieties of capacitors are commercially available at low prices thanks to mass production. The electricity storage volume density $w$ of conventional SMESs is in the sub-Wh/L range, while that of capacitors is in the Wh/L range. To increase the $w$ of SMESs to rank with or surpass that of capacitors and to produce them at a low cost by mass production, a compact SMES of a new concept produced by semiconductor microfabrication technologies has been proposed. In parallel with the experimental development, a series of trials have been carried out to estimate the feasible value of $w$ based on the calculation of the magnetic field generated by the compact SMES by improving the calculation model step by step. In the present work, (1) the applied magnetic flux density $(B)$ dependence of superconductive critical current density $\left(j_{c}\right)$ and (2) the equivalent radius of a single circular current, which generates the same $B$ at the center of the circle as the single wafer coil (engraved with a spiral coil) were taken into consideration for the first time in a series of trials. The equivalent radius was used in the calculation of the magnetic energy storage in a single-layer solenoid coil as an equivalent model of stacked wafer coils. Regarding the $B$ dependence of $j_{c}$, two kinds of previously reported experimentally obtained data of the $j_{\mathrm{c}}$ of $Y 123$ thin films under $B$ applied in parallel with the c-axis of the c-axis-oriented Y123 films were employed. The results of the estimation taught us that the $w$ of the compact SMES of the proposed concept can be in the Wh/L range or more, ranking with or surpassing those of presently available capacitors, including electric double-layer capacitors (EDLC). According to the experimental data of the $B$ dependence of $j_{\mathrm{c}}$ employed here, $j_{\mathrm{c}}$ has been measured to decrease moderately with a $B$ up to $31 \mathrm{~T}$. There appeared to be no indication of a rapid decrease in $j_{\mathrm{c}}$ due to the accession of the critical magnetic flux density $B_{\mathrm{c}}$. If a superconductivity is attained in $B>31 \mathrm{~T}$ and the measured $B-j_{\mathrm{c}}$ curve can be extrapolated beyond $31 \mathrm{~T}$, the $w$ of the compact SMES proposed here can be increased further.

Supplementary Materials: The following are available online at https: / www.mdpi.com/2312-748 1/7/3/44/s1: Figure S1: $R_{\min }$ dependence of $w, S_{\max }$ and $\Sigma B_{\max }$ for $d=50 \mu \mathrm{m}, \mathrm{s}=22 \mu \mathrm{m}, m=600$, and (a) $z=30 \mu \mathrm{m},(\mathrm{b}) z=100 \mu \mathrm{m}$ based on Equation (11). Figure S2: $R_{\min }$ dependence of $w, S_{\max }$ and $\Sigma B_{\max }$ for $d=50 \mu \mathrm{m}, s=22 \mu \mathrm{m}, m=200$, and (a) $z=30 \mu \mathrm{m}$, (b) $z=100 \mu \mathrm{m}$ based on Equation (11). Figure S3: $R_{\min }$ dependence of $w, S_{\max }$ and $\Sigma B_{\max }$ for $d=70 \mu \mathrm{m}, s=2 \mu \mathrm{m}, m=600$, and (a) $z=30 \mu \mathrm{m}$, (b) $z=100 \mu \mathrm{m}$ based on Equation (11). Figure S4: $R_{\min }$ dependence of $w, S_{\max }$ and $\Sigma B_{\max }$ for $d=70 \mu \mathrm{m}, s=2 \mu \mathrm{m}, m=200$, and (a) $\mathrm{z}=30 \mu \mathrm{m}$, (b) $\mathrm{z}=100 \mu \mathrm{m}$ based on Equation (11). Figure S5: $R_{\min }$ dependence of $w, S_{\max }$ and $\Sigma B_{\max }$ for $d=50 \mu \mathrm{m}, s=22 \mu \mathrm{m}, m=600$, and (a) $z=30 \mu \mathrm{m}$, (b) $z=100 \mu \mathrm{m}$ based on Equation (12). Figure S6: $R_{\min }$ dependence of $w, S_{\max }$ and $\Sigma B_{\max }$ for $d=50 \mu \mathrm{m}, s=22 \mu \mathrm{m}$, $m=200$, and (a) $z=30 \mu \mathrm{m}$, (b) $z=100 \mu \mathrm{m}$ based on Equation (12). Figure S7: $R_{\min }$ dependence of $w, S_{\max }$ and $\Sigma B_{\max }$ for $d=70 \mu \mathrm{m}, s=2 \mu \mathrm{m}, m=600$, and (a) $z=30 \mu \mathrm{m}$, (b) $\mathrm{z}=100 \mu \mathrm{m}$ based on Equation (12). Figure S8: $R_{\min }$ dependence of $w, S_{\max }$ and $\Sigma B_{\max }$ for $d=70 \mu \mathrm{m}, s=2 \mu \mathrm{m}, m=200$, and (a) $z=30 \mu \mathrm{m}$, (b) $z=100 \mu \mathrm{m}$ based on Equation (12).

Author Contributions: Conceptualization, T.M., M.S., and J.-h.N.; methodology, T.M., M.S., and J.-h.N.; software, T.M.; validation, T.M., M.S., and J.-h.N.; formal analysis, T.M.; investigation, T.M., M.S., and J.-h.N.; resources, T.M., M.S., and J.-h.N.; data curation, T.M.; Writing-Original draft preparation, T.M.; Writing-Review and editing, T.M., M.S., J.-h.N., and O.T.; visualization, T.M.; supervision, T.M. and O.T.; project administration, T.M.; funding acquisition, T.M., M.S., and J.-h.N. All authors have read and agreed to the published version of the manuscript.

Funding: This research was funded by a Kakenhi Grant-in-Aid for Scientific Research from the Japan Society for the Promotion of Science, grant number 19H02195.

Institutional Review Board Statement: Not applicable. 
Informed Consent Statement: Not applicable.

Data Availability Statement: Not applicable.

Acknowledgments: The authors would like to thank S. Nomura of Meiji Univ. and T. Hioki of Nagoya Univ. for their helpful discussions, and N. Sugimoto of Toyota Central R\&D Labs., Inc., for his help in the experimental procedure. The authors also thank A. Ichiki for his help in constructing the Python code for the numerical estimations and N. Saito of Nagoya University for his help in the development of the research environment.

Conflicts of Interest: The authors declare no conflict of interest.

\section{References}

1. Panasonic Electric Double Layer Capacitor (Gold Capacitor) Radial Lead Type Series: HL. Available online: https://industrial. panasonic.com/cdbs/www-data/pdf/RDH0000/ABC0000C106.pdf (accessed on 13 February 2021).

2. Nippon Chemi-Con Electric Double Layer Capacitor Dlcap ${ }^{\mathrm{tm}}$ DXE Series. Available online: https://www.chemi-con.co.jp/ catalog/pdf/dl-je/dl-sepa-je/dl-dxe-je-2020.pdf (accessed on 13 February 2021).

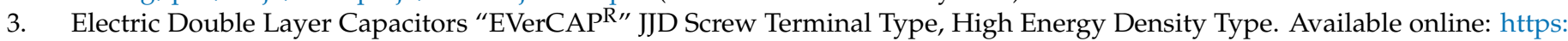
/ / www.nichicon.co.jp/english/products/evercap/index.html (accessed on 13 February 2021).

4. VISHAY 196 HVC ENYCAPTM Hybrid Energy Storage Capacitors. Available online: https://www.vishay.com/docs/28409/19 6hvc.pdf (accessed on 13 February 2021).

5. Venkataratum, K.; Rao, V.V.; Rao, K.N.V.S.; Kumar, A.A. Optimurn Design of Superconducting Magnet Coil for a Micro SMES Unit. IEEE Trans. Appl. Supercond. 1999, 9, 350-353. [CrossRef]

6. Shikimachi, K.; Hirano, N.; Nagaya, S.; Kawashima, H.; Higashikawa, K.; Nakamura, T. System Coordination of 2 GJ Class YBCO SMES for Power System Control. IEEE Trans. Appl. Supercond. 2009, 19, 2012-2018. [CrossRef]

7. Katagiri, T.; Nakabayashi, H.; Nijo, Y.; Tamada, T.; Noda, T.; Hirano, N.; Nagaya, T.; Nagaya, S. Field Test Result of 10MVA/20MJ SMES for Load Fluctuation Compensation. IEEE Trans. Appl. Supercond. 2009, 19, 1993-1998. [CrossRef]

8. Zimmermann, A.W.; Sharkh, S.M. Design of a $1 \mathrm{MJ} / 100 \mathrm{~kW}$ high temperature superconducting magnet for energy storage. Energy Rep. 2020, 6, 180-188. [CrossRef]

9. Kumar, A.; Jeyan, J.V.M.L.; Agarwal, A. Electromagnetic Analysis on 2.5 MJ High Temperature Superconducting Magnetic Energy Storage (SMES) Coil to be used in Uninterruptible Power Applications. Mater. Today Proc. 2020, 21, 1755-1762. [CrossRef]

10. Zhang, J.Y.; Song, N.H.; Gao, Z.Y.; Zhang, F.Y.; Jing, L.W.; Guo, W.Y.; Teng, Y.P.; Zhang, R.L.; Xin, Z.Z.; Zhang, D.; et al. Manufacture and Tests of a Bi2223/YBCO Coil for a 1-MJ/0.5MVA Fault Current Limitter-Magnetic Energy Storage System. J. Supercond. Novel Mag. 2019, 32, 521-528. [CrossRef]

11. Chen, Z.; Geng, G.; Fang, J. Influence of AC Loss on Stress and Strain of Superconducting Coils. J. Supercond. Novel Magn. 2019, 32, 549-555. [CrossRef]

12. Vyas, G.; Dondapai, R.S. Investigation on the structural behavior of superconducting magnetic energy storage (SMES) devices. J. Energy Storage 2020, 28, 101212. [CrossRef]

13. Kumar, A.; Muruga Lal Jeyan, J.V.; Agarwal, A. Numerical analysis on 10MJ solenoidal high temperature superconducting magnetic energy storage system to evaluate magnetic flux and Lorentz force distribution. Phys. C Amst. Neth. 2019, 558, 17-24. [CrossRef]

14. Watanabe, T.; Nagaya, S.; Hirano, N.; Awaji, S.; Oguro, H.; Ishiyama, A.; Hojo, M.; Nishikawa, M. Progress of “Yoroi-Coil Structure" in Mechanical Strength with High Current Density. IEEE Trans. Appl. Supercond. 2017, 27, 4602305. [CrossRef]

15. Nishijima, G.; Oguro, H.; Awaji, S.; Watanabe, K.; Shikimachi, K.; Hirano, N.; Nagaya, S. Transport Characteristics of CVD-YBCO Coated Conductor under Hoop Stress. IEEE Trans. Appl. Supercond. 2008, 18, 1131-1134. [CrossRef]

16. Morandi, A.; Trevisani, L.; Negrini, F.; Ribani, P.L.; Fabbri, M. Feasibility of Superconducting Magnetic Energy Storage on Board of Ground Vehicles with Present State-of-the-Art Superconductors. IEEE Trans. Appl. Supercond. 2012, 22, 5700106. [CrossRef]

17. Ko, C.-T.; Chen, K.-N. Wafer-level bonding/stacking technology for 3D integration. Microelectron. Reliab. 2010, 50, 481-488. [CrossRef]

18. Sumitomo Heavy Industries, Ltd. Cryocoolers. Available online: https://www.shi.co.jp/english/products/precision/cold/ index.html (accessed on 13 February 2021).

19. Sugimoto, N.; Motohiro, T. Anisotropic I-V characteristics of spontaneously emerged periodic stripes of superconducting NbN thin films on Si trench sidewall by RF magnetron sputtering. Vacuum 2012, 93, 13-21. [CrossRef]

20. Sugimoto, N.; Iguchi, N.; Kusano, Y.; Fukano, T.; Hioki, T.; Ichiki, A.; Bessho, T.; Motohiro, T. Compact SMES with a superconducting film in a spiral groove on a Si wafer formed by MEMS technology with possible high-energy storage volume density comparable to rechargeable batteries. Supercond. Sci. Technol. 2017, 30, 015014. [CrossRef]

21. Sasaki, M.; Hsu, C.-W.; Suzuki, Y.; Hioki, T.; Noh, J.-H.; Takai, O.; Watanabe, H.; Doy, H.; Motohiro, T. Fabrication of 3-stepped spiral trench with smooth sidewall at nano-level to deposit superconducting material for energy storage. Int. J. Nanotechnol. 2018, 15, 858-872. [CrossRef] 
22. Wang, M.; Li, J.; Ngo, K.; Xie, H. Silicon molding techniques for integrated power MEMS inductors, Sens. Actuators A 2011, 166, 157-163. [CrossRef]

23. Suzuki, Y.; Iguchi, N.; Adachi, K.; Hioki, T.; Ichiki, A.; Hsu, C.-W.; Kumagai, S.; Sasaki, M.; Motohiro, T. Stress control of reactively sputtered thick $\mathrm{NbN}$ film on $\mathrm{Si}$ wafer changing the location of the substrate $\mathrm{Si}$ wafer against the $\mathrm{Nb}$ target on a magnetron cathode. IOP Conf. Series J. Phys. Conf. Series 2017, 871, 012071. [CrossRef]

24. Suzuki, Y.; Iguchi, N.; Adachi, K.; Ichiki, A.; Hioki, T.; Hsu, C.-W.; Sato, R.; Kumagai, S.; Sasaki, M.; Noh, J.-H.; et al. Complete fabrication of a traversable $3 \mu \mathrm{m}$ thick $\mathrm{NbN}$ film superconducting coil with Cu plated layer of $42 \mathrm{~m}$ in length in a spiral threestoried trench engraved in a Si wafer of $76.2 \mathrm{~mm}$ in diameter formed by MEMS technology for a compact SMES with high energy storage volume density. IOP Conf. Ser. J. Phys. Conf. Ser. 2017, 897, 012019. [CrossRef]

25. Manabe, T.; Kondo, W.; Mizuta, S.; Kumagai, T. Cristallization of $\mathrm{YBa}_{2} \mathrm{Cu}_{3} \mathrm{O}_{7-\mathrm{y}}$ films on $\mathrm{SrTiO}_{3}(100)$ by postannealing of precursors prepared by dipping-pyrolysis process. J. Mater. Res. 1994, 9, 858-865. [CrossRef]

26. Motoki, T.; Ikeda, S.; Honda, G.; Nagaishi, T.; Nakamura, S.; Shimoyama, J. Dramatic effects of chlorine addition on expanding synthesis conditions for fluorine-free metal-organic decomposition YBa2Cu3Oy films. Appl. Phys. Express 2017, 10, 023102. [CrossRef]

27. Ichiki, Y.; Adachi, K.; Suzuki, Y.; Kawahara, M.; Ichiki, A.; Hioki, T.; Hsu, C.-W.; Kumagai, S.; Sasaki, M.; Noh, J.-H.; et al. Replacement of $\mathrm{NbN}$ by $\mathrm{YBa}_{2} \mathrm{Cu}_{3} \mathrm{O}_{7-\delta}$ in superconducting thin film coil in a spiral trench on a Si-wafer for compact SMESs. IOP Conf. Ser. J. Phys. Conf. Ser. 2018, 1054, 012065. [CrossRef]

28. National High Magnetic Field Laboratory. Data of YBCO Tape, Magnetic Field Perpendicular to the Tape-Plane. REBCO: SP26 Tape, $50 \mu \mathrm{m}$ Substrate, 7.5\% Zr. Measured at National High Magnetic Field Laboratory (NHMFL) by V. Braccini, J. Jaroszynski and A. Xu. 2007. Available online: https://nationalmaglab.org/magnet-development/applied-superconductivity-center/ (accessed on 13 February 2021).

29. Braccini, V.; Xu, A.; Jaroszynski, J.; Xin, Y.; Larbalestier, D.C.; Chen, Y.; Carota, G.; Dackow, J.; Kesgin, I.; Yao, Y.; et al. Properties of recent IBAD-MOCVD coated conductors relevant to their high field, low temperature magnet use. Supercond. Sci. Technol. 2010, 24, 035001. [CrossRef]

30. Slideum Directory Jc vs. B-Florida State University. Data of YBCO: /Ni/YSZ 1 $\mu$ m Thick Microbridge, Magnetic Field in Parallel with c-Axis of the YBCO Thin Film, 4 K, Foltyn et al. (LANL) '96. Available online: https://slideum.com/doc/1756013/ jc-vs-b---florida-state-university (accessed on 13 February 2021).

31. Sekitani, T.; Miura, N.; Ikeda, S.; Matsuda, Y.H.; Shiobara, Y. Upper critical field for optimally-doped $\mathrm{YBa}_{2} \mathrm{Cu}_{3} \mathrm{O}_{7-\delta}$. Phys. B Amst. Neth. 2004, 346/347, 319-324. [CrossRef]

32. Ichiki, Y.; Ichiki, A.; Hioki, T.; Sasaki, M.; Noh, J.-H.; Takai, O.; Honma, H.; Motohiro, T. Estimation of electricity storage capacity of compact SMESs composed of stack of Si-wafers loaded with superconducting thin film coils in spiral trenches formed by MEMS process. IOP Conf. Ser. J. Phys. Conf. Ser. 2019, 1293, 012058. [CrossRef]

33. Motohiro, T.; Sasaki, M.; Noh, J.-H.; Takai, O.; Honma, H. Estimation of Electricity Storage Density of Compact SMESs Composed of Si-wafer Stacks Loaded with Superconducting Thin Film Coils in Spiral Trenches under the Constraints of the Critical Magnetic Flux Density, J. Phys. Conf. Ser. 2020, 1590, 012045. [CrossRef]

34. Rombolà, G.; Ballarini, V.; Chiodoni, A.; Gozzelino, L.; Mezzetti, E.; Menetti, B.; Pirri, C.F.; Tresso, E.R.F. Sputtering deposition of buffer layers for Si/YBCO integrated microelectronics. Int. J. Mod. Phys. B 2005, 19, 4605-4617. [CrossRef]

35. Botta, D.; Camerlingo, C.; Chiodoni, A.; Fabbri, F.; Gerbaldo, R.; Chigo, G.; Gozzelino, L.; Laviano, F.; Minetti, B.; Pirri, C.F.; et al. Intrinsic pinning and current percolation signatures in E-J characteristics of $\mathrm{Si} / \mathrm{YSZ} / \mathrm{CeO}_{2} / \mathrm{YBCO}$ layouts. Eur. Phys. J. B 2005, 48, 359-365. [CrossRef]

36. Shimadzu Europa GmbH. Press Information 2018, New Nexgen GC CAGC-100, A-ENG-18008. 10 April 2018. Available online: https:/ / www.shimadzu.eu/new-nexgen-gc-cagc-100 (accessed on 13 February 2021).

37. Nishino, M.; Takemori, Y.; Matsuoka, S.; Kanai, M.; Nishimoto, T.; Ueda, M.; Komori, K. Development of $\mu$ GC (Micro Gas Chromatography) with High Performance Micromachined Chip Column. IEEJ Trans. Electr.Electron. Eng. 2009, 4, 358-364. [CrossRef]

38. Sekiya, N.; Monjunaga, Y. A novel REBCO wire structure that improves coil quality factor in $\mathrm{MHz}$ range and its effect on wireless power transfer system. IEEE Trans. Appl. Supercond. 2017, 27, 6602005. [CrossRef]

39. Nomura, S.; Tsutsui, H.; Shimada, R. Feasibility Study on Large Scale SMES for Daily Load Leveling Using Force-Balanced Helical Coils. IEEE Trans. Appl. Supercond. 2013, 23, 5700904. [CrossRef]

40. Penthia, T.; Panda, A.K.; Patnaik, N.; Mohanty, P.R. Performance of SMES system with non-linear dynamic control approach for pulsed load compensation. IET Gener. Transm. Distrib. 2020, 14, 1872-1881. [CrossRef]

41. NTT R\&D Forum 2020 Connect E03_j. Available online: https://www.rd.ntt/_assets/pdf/forum/2020/E03_j.pdf (accessed on 13 February 2021). 